\title{
Parametrization and calibration of a quasi-analytical algorithm for tropical eutrophic waters
}

\author{
Fernanda Watanabe ${ }^{a}$, Deepak R. Mishra ${ }^{b}$, Ike Astuti ${ }^{b}$, Thanan Rodrigues ${ }^{a}$, Enner Alcântara ${ }^{a, *}$, \\ Nilton N. Imai ${ }^{a}$, Cláudio Barbosa ${ }^{c}$ \\ ${ }^{a}$ Department of Cartography, Sao Paulo State University, Presidente Prudente, SP, Brazil \\ ${ }^{\mathrm{b}}$ Center for Geospatial Research, Department of Geography, University of Georgia, Athens, GA, USA \\ ${ }^{\mathrm{c}}$ Image Processing Division, National Institute for Space Research, São José dos Campos, SP, Brazil
}

\section{A R T I C L E I N F O}

\section{Article history:}

Received 11 May 2016

Received in revised form 8 August 2016

Accepted 18 August 2016

Available online 29 September 2016

\section{Keywords:}

Quasi-analytical algorithm

Inland waters

Algal bloom

Bio-optical model

Remote sensing reflectance

Inherent optical properties

\begin{abstract}
A B S T R A C T
Quasi-analytical algorithm (QAA) was designed to derive the inherent optical properties (IOPs) of water bodies from above-surface remote sensing reflectance $\left(R_{r s}\right)$. Several variants of QAA have been developed for environments with different bio-optical characteristics. However, most variants of QAA suffer from moderate to high negative IOP prediction when applied to tropical eutrophic waters. This research is aimed at parametrizing a QAA for tropical eutrophic water dominated by cyanobacteria. The alterations proposed in the algorithm yielded accurate absorption coefficients and chlorophyll- $a$ (Chl- $a$ ) concentration. The main changes accomplished were the selection of wavelengths representative of the optically relevant constituents (ORCS) and calibration of values directly associated with the pigments and detritus plus colored dissolved organic material (CDM) absorption coefficients. The re-parametrized QAA eliminated the retrieval of negative values, commonly identified in other variants of QAA. The calibrated model generated a normalized root mean square error (NRMSE) of $21.88 \%$ and a mean absolute percentage error (MAPE) of $28.27 \%$ for $a_{t}(\lambda)$, where the largest errors were found at $412 \mathrm{~nm}$ and $620 \mathrm{~nm}$. Estimated NRMSE for $a_{C D M}(\lambda)$ was $18.86 \%$ with a MAPE of $31.17 \%$. A NRMSE of $22.94 \%$ and a MAPE of $60.08 \%$ were obtained for $a_{\varphi}(\lambda)$. Estimated $a_{\varphi}(665)$ and $a_{\varphi}(709)$ was used to predict Chl- $a$ concentration. $a_{\varphi}(665)$ derived from QAA for Barra Bonita Hydroelectric Reservoir (QAA_BBHR) was able to predict Chl- $a$ accurately, with a NRMSE of $11.3 \%$ and MAPE of $38.5 \%$. The performance of the Chl-a model was comparable to some of the most widely used empirical algorithms such as 2-band, 3-band, and the normalized difference chlorophyll index (NDCI). The new QAA was parametrized based on the band configuration of MEdium Resolution Imaging Spectrometer (MERIS), Sentinel-2A and 3A and can be readily scaled-up for spatiotemporal monitoring of IOPs in tropical waters.
\end{abstract}

(c) 2016 International Society for Photogrammetry and Remote Sensing, Inc. (ISPRS). Published by Elsevier B.V. All rights reserved.

\section{Introduction}

Proximal and satellite remote sensing for marine and freshwater systems is often aimed at estimating the optically relevant constituents (ORCs) such as phytoplankton, total suspended sediments (TSS), and colored dissolved organic matter (CDOM) concentrations from remote sensing reflectance $\left(R_{r s}\right)$. $R_{r s}$ is directly related to the inherent optical properties (IOPs) of the water, i.e., absorption ( $a$ ) and backscattering coefficients $\left(b_{b}\right)$ as described by Gordon et al. (1988):

\footnotetext{
* Corresponding author.

E-mail address: ennerha@gmail.com (E. Alcântara).
}

$$
R_{r s}(\lambda) \propto \frac{b_{b}(\lambda)}{b_{b}(\lambda)+a(\lambda)}
$$

where $a(\lambda)$ is the absorption coefficients of phytoplankton, detritus, $\mathrm{CDOM}$, and pure water and $b_{b}(\lambda)$ is represented by the sum of backscattering of particulate material and pure water. Table 1 lists all symbols, abbreviations and definitions used in this study.

Marine and freshwater systems contain a differing variety of particles and dissolved substances and the variability in absorption and backscattering properties associated with these constituents hampers the extraction of quantitative information about them (Morel and Prieur, 1977). Several remote sensing methods have been developed to quantify ORCs responsible for water color using statistical regressions (Vincent et al., 2004; Mishra and Mishra, 
Table 1

Symbols, abbreviations, and definitions.

\begin{tabular}{|c|c|c|}
\hline Symbols & Definition & Unit \\
\hline$a(\lambda), a_{t}(\lambda)$ & Absorption coefficient of the total, $a_{\mathrm{CDM}}+a_{\varphi}+a_{w}$ & $\left(\mathrm{~m}^{-1}\right)$ \\
\hline$a_{p}(\lambda)$ & Absorption coefficient of particulate material & $\left(\mathrm{m}^{-1}\right)$ \\
\hline$a_{d}(\lambda)$ & Absorption coefficient of detritus & $\left(\mathrm{m}^{-1}\right)$ \\
\hline$a_{\text {CDOM }}(\lambda)$ & Absorption coefficient of colored dissolved organic material & $\left(\mathrm{m}^{-1}\right)$ \\
\hline$a_{C D M}(\lambda)$ & Absorption coefficient of detritus and CDOM & $\left(\mathrm{m}^{-1}\right)$ \\
\hline$a_{\varphi}(\lambda)$ & Absorption coefficient of phytoplankton pigments & $\left(\mathrm{m}^{-1}\right)$ \\
\hline$a_{w}(\lambda)$ & Absorption coefficient of pure water & $\left(\mathrm{m}^{-1}\right)$ \\
\hline$a_{t-w}(\lambda)$ & Absorption coefficient of the total minus pure water & $\left(\mathrm{m}^{-1}\right)$ \\
\hline ORC & Optically relevant constituent & \\
\hline$b_{b}(\lambda)$ & Backscattering of the total, $b_{b p}+b_{w}$ & $\left(\mathrm{~m}^{-1}\right)$ \\
\hline$b_{b p}(\lambda)$ & Backscattering of particle & $\left(\mathrm{m}^{-1}\right)$ \\
\hline$b_{w}(\lambda)$ & Backscattering of pure water & $\left(\mathrm{m}^{-1}\right)$ \\
\hline BBHR & Barra Bonita hydroelectric reservoir & \\
\hline CDOM & Colored dissolved organic matter & \\
\hline CDM & Colored detrital material & \\
\hline Chl- $a$ & Chlorophyll- $a$ & $\left(\mathrm{mg} \mathrm{m}^{-3}\right)$ \\
\hline IOP & Inherent optic properties & \\
\hline$\eta$ & Spectral power for particle backscattering coefficient & \\
\hline$R_{r s}(\lambda)$ & Above surface remote sensing reflectance & $\left(\mathrm{sr}^{-1}\right)$ \\
\hline$r_{r s}(\lambda)$ & Below surface remote sensing reflectance & $\left(\mathrm{sr}^{-1}\right)$ \\
\hline$S$ & Spectral slope for detritus and CDOM absorption coefficient & \\
\hline$u$ & Ratio of backscattering coefficient to the sum of absorption and backscattering coefficient, $b_{b} /\left(a+b_{b}\right)$ & \\
\hline$\lambda_{0}$ & Reference wavelength & $(\mathrm{nm})$ \\
\hline$\zeta$ & $a_{\varphi}(411) / a_{\varphi}(443)$ & \\
\hline$\xi$ & $a_{C D M}(411) / a_{C D M}(443)$ & \\
\hline
\end{tabular}

2010; Gurlin et al., 2011; Odermatt et al., 2012; Kumar et al., 2016) and inversion algorithm techniques (Hoge and Lyons, 1996; Brando and Dekker, 2003; Doerffer and Schiller, 2007; Odermatt et al., 2012). These models are based on $R_{r s}$ or irradiance reflectance $(R)$ and use empirical, semi-analytical and quasi-analytical approaches.

Empirical models are based on statistical regression between water properties and $R_{r s}$ or $R$ measurements. These models do not utilize the IOPs which limits their applicability in terms of temporal and geographic spread (Moses et al., 2012; Odermatt et al., 2012; Lee et al., 2002). Semi-analytical models involve numerical optimization and are based on solutions for radiative transfer equations (Hoge and Lyons, 1996; Brando and Dekker, 2003; Lee et al., 2002; Chen et al., 2014). Nevertheless, they require some empirical solution (Odermatt et al., 2012). These models have the geographic and temporal flexibility and can be applied to other aquatic environments (Lee et al., 2002). However, their performances depend on the parameterization to be representative of the IOPs of that environment (Lee et al., 2002).

The quasi-analytical algorithm (QAA) was originally developed by Lee et al. (2002) to derive $a(\lambda)$ and $b_{b}(\lambda)$ from $R_{r s}$ directly below the air-sea interface $\left(r_{r s}\right)$. Derived $a(\lambda)$ are decomposed to absorption by phytoplankton $\left(a_{\varphi}(\lambda)\right)$ and CDOM plus detritus (CDM) $\left(a_{C D M}(\lambda)\right)$, whereas, $b_{b}(\lambda)$ is estimated from the backscattering coefficient for particulate material $\left(b_{b p}\right)$ (Lee et al., 2002, 2009; Mishra et al., 2013, 2014; Lee, 2014). Lee et al. (2002) showed that QAA presented similar accuracy when compared to approaches using optimization techniques. Several versions of QAA have been parametrized and tested for aquatic environments with different IOPs such as open ocean and coastal waters (Lee et al., 2002, 2009; IOCCG, 2006; Lee, 2014; Wei et al., 2015; Chen and Zhang, 2015), lakes (Le et al., 2009), rivers (Zhu et al., 2013; Li et al., 2013, 2015), and ponds (Mishra et al., 2013, 2014).

Wavelength reference $\left(\lambda_{0}\right)$ and the spectral power for particle backscattering coefficient $(\eta)$ have been appointed as the main source of errors in estimating $a\left(\lambda_{0}\right)$ (Le et al., 2009; Yang et al., 2013). The selection of the $\left(\lambda_{0}\right)$ is essential for an accurate performance of the QAA and must be dominated by pure water absorp- tion $\left(a_{w}\right)$. QAA was originally developed for open ocean waters by Lee et al. (2002) using $555 \mathrm{~nm}$ as $\lambda_{0}$. The algorithm was parametrized and calibrated using ocean $R_{r s}$ data available from NASA bio-Optical Marine Algorithm Dataset (NOMAD) (SeaBASS, 2015). Lee and Carder (2004) applied the native QAA to coastal waters, with chlorophyll- $a$ (Chl- $a$ ) concentration ranging from 0.16 to $11.3 \mathrm{mg} \mathrm{m}^{-3}$. Other versions up to version 5 , all developed for marine environments, adopted $555 \mathrm{~nm}$ as $\lambda_{0}$ (Lee et al., 2009). Lee (2014) proposed $667 \mathrm{~nm}$ as $\lambda_{0}$ for waters with $R_{r s}(670)>0.0065 \mathrm{sr}^{-1}$, i.e., waters with higher concentrations of Chl-a.

In inland waters, there is a high influence of $a_{\varphi}(\lambda)$ and $a_{C D M}(\lambda)$ over $a(\lambda)$ at shorter wavelengths and hence other authors (Le et al., 2009; Mishra et al., 2013, 2014; Li et al., 2013, 2015) have shifted $\lambda_{0}$ toward the red-edge region, improving significantly the estimation of $a\left(\lambda_{0}\right)$. Le et al. (2009) conducted a study in Taihu Lake, a highly turbid eutrophic water body. They used the wavelength at $710 \mathrm{~nm}$ as $\lambda_{0}$. Mishra et al. $(2013,2014)$ developed a version for highly productive waters with a Chl- $a$ range of $59.40-1376.60 \mathrm{mg} \mathrm{m}^{-3}$ and TSS range of $69.80-401.20 \mathrm{~g} \mathrm{~m}^{-3}$. They did not immediately observed an improvement in the model performance by shifting $\lambda_{0}$ to $708 \mathrm{~nm}$, because there still was influence of particles absorption at this wavelength. Several attempts were carried out until they found a satisfactory empirical relationship between $a$ and $r_{r s}$.

Li et al. $(2013,2015)$ also developed QAA versions for inland waters. The first model developed by Li et al. (2013) was parametrized for an environment with a Chl- $a$ range of 1.85$285.8 \mathrm{mg} \mathrm{m}^{-3}$ and TSS range of $1.51-211.91 \mathrm{~g} \mathrm{~m}^{-3}$. The second model was a QAA for an environment with a Chl- $a$ range of $2.93-285.8 \mathrm{mg} \mathrm{m}^{-3}$ and TSS of $2.34-123.79 \mathrm{~g} \mathrm{~m}^{-3}$. They changed the structure of some QAA steps while using $709 \mathrm{~nm}$ as $\lambda_{0}$. Yang et al. (2013) developed a study in three turbid Asian lakes with a Chl- $a$ range of $9.79-153.92 \mathrm{mg} \mathrm{m}^{-3}$ and TSS range of 4.81$61.00 \mathrm{~g} \mathrm{~m}^{-3}$. They shifted the position of $\lambda_{0}$ to $753 \mathrm{~nm}$ to prevent the interference of the high turbidity over $a\left(\lambda_{0}\right)$.

Bio-optical status of the water body at these locations is very different from each other, therefore, these versions may not be 
directly applicable to all aquatic systems with widely varying range of OACs. Our study area exhibited a Chl- $a$ range of 17.7$797.8 \mathrm{mg} \mathrm{m}^{-3}$ and TSS range of $3.6-44 \mathrm{~g} \mathrm{~m}^{-3}$. This extreme Chl- $a$ range is outside the calibration range of all the aforementioned QAAs and therefore, produces high errors in IOP prediction. The objective of this study were to parametrize and calibrate a QAA to retrieve IOPs and use them in a bio-optical model for estimating Chl- $a$ concentration in a tropical eutrophic reservoir with extreme variability in phytoplankton biomass.

The newly re-parametrized QAA is based on the QAA_v5 proposed by Lee et al. (2009). Radiometric data and water samples collected in situ were used to parametrize and calibrate the empirical steps (Lee et al., 2002) of QAA. Alterations to some empirical steps considerably improved the retrieval of $a_{t}(\lambda), a_{C D M}(\lambda)$ and $a_{\varphi}(\lambda)$. The critical alterations as part of the re-parameterization were (a) the selection the wavelengths suitable for the OACs composition; (b) calibration of $a\left(\lambda_{0}\right)$; (c) parameterization and calibration of $\zeta$ that is a value associated with $a_{\varphi}$; and (d) calibration of $\xi$, the parameter associated with $a_{C D M}$. The accurate calibration and parameterization of $a_{t-w}\left(\lambda_{0}\right)$ was essential to derive $a\left(\lambda_{0}\right)$ and other IOPs, being that the wavelengths associated with absorption by Chl- $a$ and phycocyanin (PC) $(443,620$ and $665 \mathrm{~nm})$ were the most appropriate. The novelty of this research is related to estimation of $a_{C D M}(\lambda)$ and $a_{\varphi}(\lambda)$, which were underestimated with all existing QAA, yielding negative values. The fit of CDOM spectral slope $(S)$ improved the accuracy of algorithm, but the alterations carried out in $\zeta$ were the key contributions of this study. This new variant of QAA would be applicable to tropical reservoirs exhibiting eutrophic to hypereutrophic conditions.

\section{Data and methods}

\subsection{Study area}

Barra Bonita hydroelectric reservoir (BBHR) $\left(22^{\circ} 31^{\prime} 10^{\prime \prime} \mathrm{S}\right.$ and $48^{\circ} 32^{\prime} 3^{\prime \prime} \mathrm{W}$ ) lies in the middle course of the Tietê River, São Paulo State, Brazil (Fig. 1). The BBHR is situated in a transitional region between tropical and subtropical climate, characterized by a dry period (May-October) and a wet period (November-April). The BBHR is the first of six reservoirs cascading in the Tietê River and was built in 1963 , flooding an area of $310 \mathrm{~km}^{2}$ with a volume of
$3.622 \times 10^{6} \mathrm{~m}^{3}$. It is a storage reservoir, presenting a minimum quota of $439.5 \mathrm{~m}$ and maximum of $451.5 \mathrm{~m}$ (AES Tietê, 2013).

According to Tundisi et al. (2008), the maximum depth of the reservoir is $25 \mathrm{~m}$, with an average of $10.2 \mathrm{~m}$. The water retention time varies from 30 days (austral summer) to 180 days (austral winter). The flow range is $1500 \mathrm{~m}^{3} \mathrm{~s}^{-1}$ in the austral summer (wet season) to $200 \mathrm{~m}^{3} \mathrm{~s}^{-1}$ in the austral winter (dry season). BBHR is characterized as eutrophic waters with high species richness and concentration of phytoplankton (Calijuri and Santos, 1996; Dellamano-Oliveira et al., 2008). The dominant species of phytoplankton in BBHR are Microcystis aeruginosa and free cells of Microcystis sp. (Cyanophyceae), and Aulacoseira granulata filaments (Bacillariophyceae). Their variation in space and time are mainly associated with water column mixing events and residence time.

The calibration data were collected in the Austral Autumn (May 5-9, 2014) and Austral Spring (October 13-16, 2014), whereas, the validation dataset was acquired in the Austral Winter (September 13-15, 2015). Water samples and radiometric measurements were collected from 20 sampling locations randomly distributed in BBHR (see Fig. 1 for location), selected using a method proposed by Rodrigues et al. (2016). Water samples were collected to estimate Chl- $a$ and TSS concentration, and IOPs (Watanabe et al., 2015).

\subsection{Remote sensing reflectance}

Radiometric measurements collected in situ were used to calculate $R_{r s}$ using Mobley (1999).

$R_{r s}=\frac{L_{w}}{E_{s}}=\frac{\left(L_{t}-\rho L_{s}\right)}{E_{s}}$

where $L_{s}$ is the incident sky radiance; $L_{t}$ is the total radiance measured above surface and composed of the water-leaving radiance $\left(L_{w}\right)$ and the portion of the $L_{s}$ that is reflected by water surface $\left(L_{r}\right) ; E_{s}$ is the incident sky irradiance; $\rho$ is a reflectance factor related to direction, wavelength, wind speed, sensor field of view (FOV) taken equal to 0.028, and sky radiance distribution (Mobley, 1999).

Acquisition geometry was adopted from Mobley (1999) and Mueller (2003). Three spectroradiometers, one ACC-VIS RAMSES with cosine collector and two ARC-VIS RAMSES with a $7^{\circ}$ fieldof-view (TriOS, Oldenburg, Germany), were used to acquire the radiometric measurements. The ACC-VIS sensor was pointed
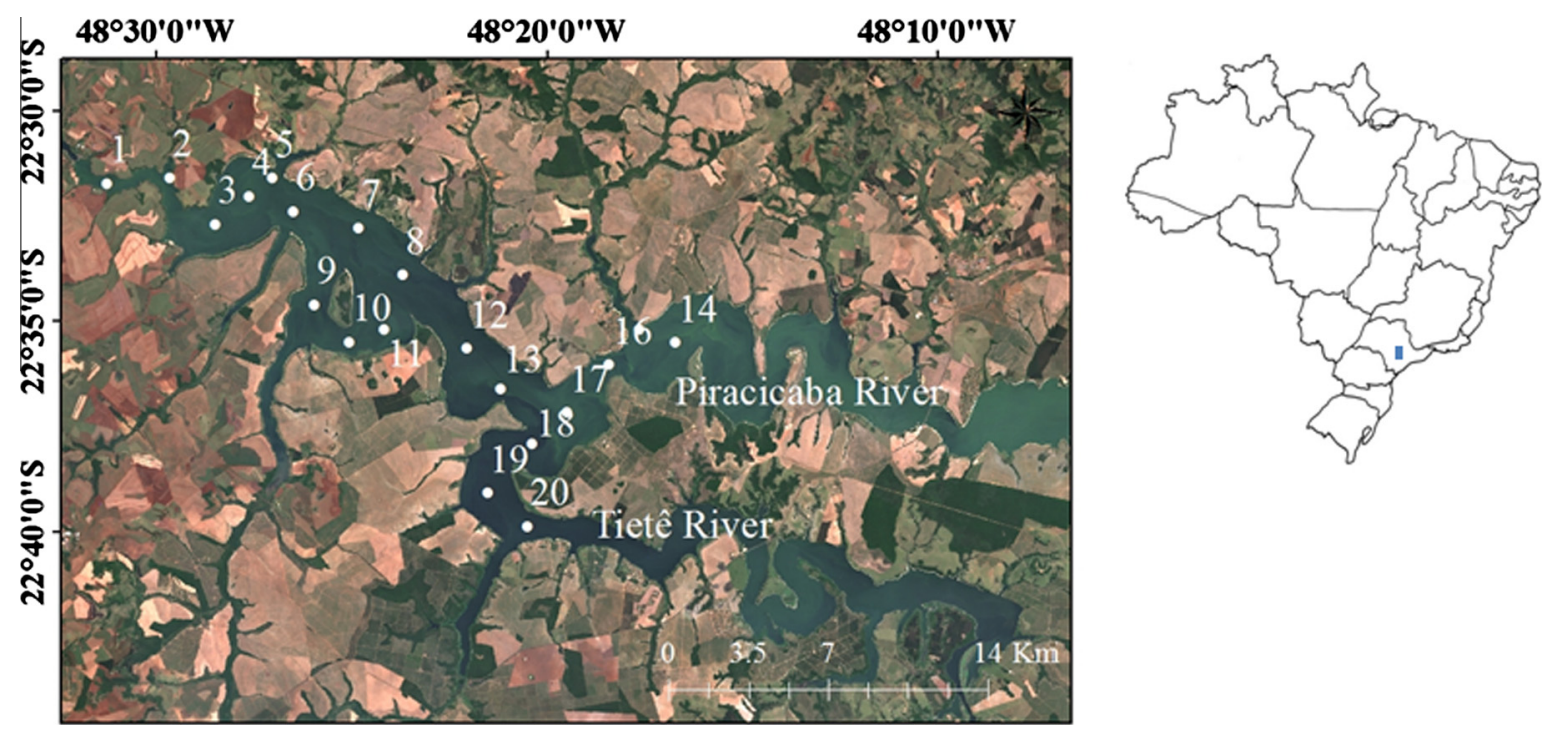

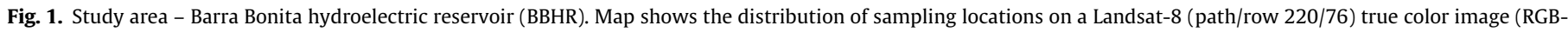
432) acquired on October 13, 2014. 

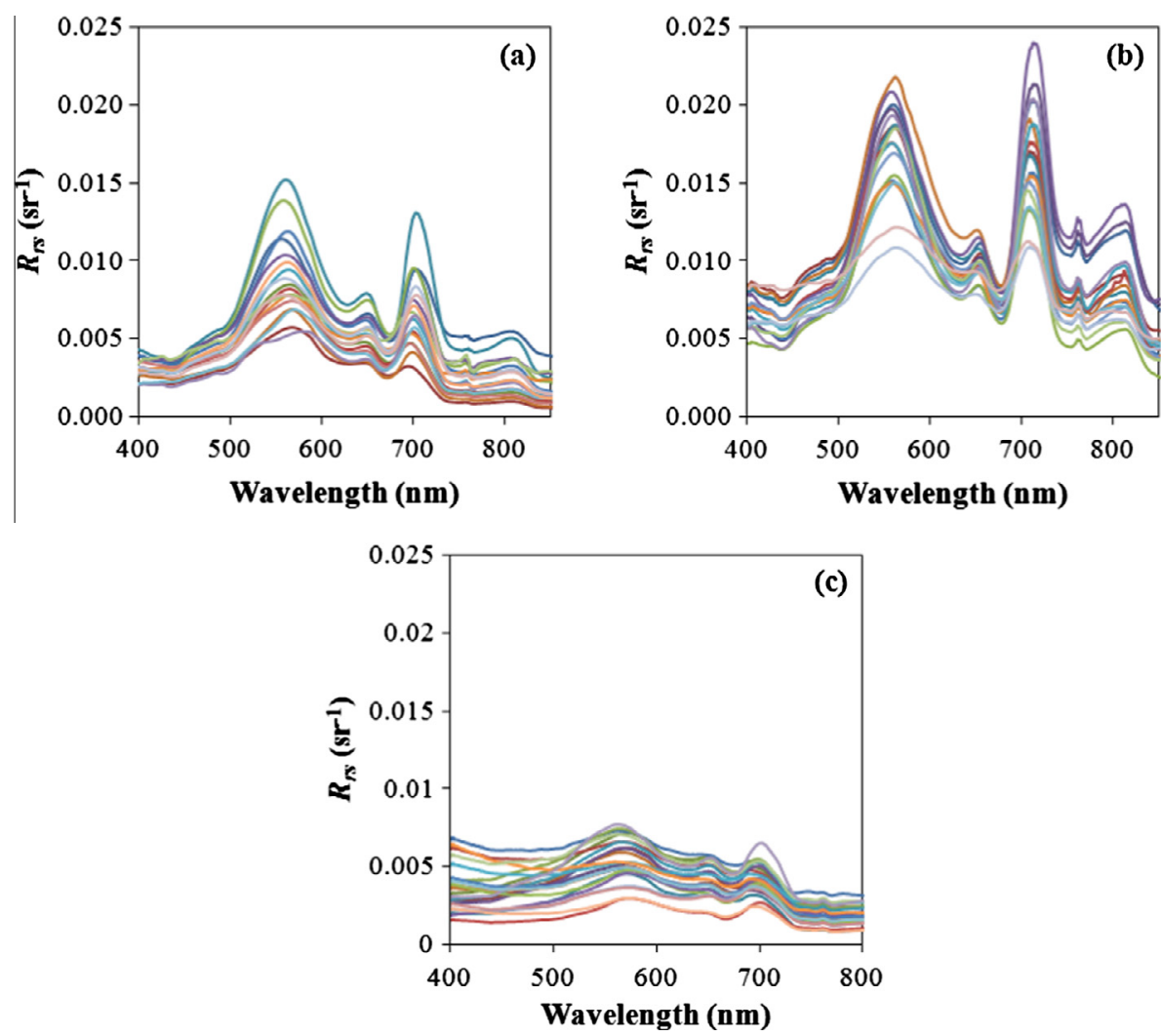

Fig. 2. In situ $R_{r s}$ spectra collected in (a) May 2014 ( $\left.n=18\right)$, (b) October $2014(n=20)$, and (c) September 2015 ( $\left.n=24\right)$.

upward to collect $E_{s}(\lambda)\left(\mathrm{W} \mathrm{m}^{-2}\right)$. One ARC-VIS sensor was pointed in downward direction (water surface) with an angle of $45^{\circ}$ in relation to nadir position of the zenith angle to measure the $L_{t}(\lambda)$ $\left(\mathrm{W} \mathrm{m}^{-2} \mathrm{sr}^{-1}\right)$. Another ARC-VIS sensor was pointed upward direction (sky) with an angle of $135^{\circ}$ in relation to nadir position of the zenith angle to measure $L_{s}(\lambda)\left(\mathrm{W} \mathrm{m}^{-2} \mathrm{sr}^{-1}\right)$. The radiometers acquired data in a wavelength range of $320-950 \mathrm{~nm}$ and a spectral sampling of approximately $3.3 \mathrm{~nm}$, therefore, all of the $E_{s}(\lambda), L_{t}(\lambda)$ and $L_{s}(\lambda)$ measurements were interpolated to $1 \mathrm{~nm}$.

Eighteen samples were collected in May 2014, 20 samples in October 2014 and 24 samples in September 2015. Fig. 2 shows the $R_{r s}$ spectra measured in the three field surveys. The 38 measurements of $R_{r s}$ (first two field surveys) were used as input to parametrize the QAA. The absorption feature of the phycocyanin (PC) pigment at approximately $620 \mathrm{~nm}$ (Weaver and Wrigley, 1994; Mishra et al., 2009, 2014; Ogashawara et al., 2013) associated with presence of cyanobacteria is clearly identified in both field campaigns. The high absorption at about $680 \mathrm{~nm}$ and reflectance at $710 \mathrm{~nm}$ is associated with high concentrations of Chl- $a$ indicating the eutrophic status of the study site. Central wavelengths of the bands available in the MEdium Resolution Imaging Spectrometer (MERIS) sensor, onboard ENVISAT-1 satellite (ESA, 2015), were adopted as reference to parametrize the QAA. Although MERIS is non-operational, the results obtained in this research can be compared with MERIS based published literature. In addition, MERIS bands are compatible with of other operational sensor systems such as MSI/Sentinel-2A, launched in June 23rd 2015, and OLCI/Sentinel-3A launched on February 16th 2016 (ESA, 2015).

\subsection{Optically relevant constituents (ORCS)}

Water samples were collected in situ to measure Chl-a, TSS, inorganic suspended solids (ISS) and organic suspended solids
(OSS) concentrations. $250 \mathrm{ml}$ water was filtered through each glass fiber $\mathrm{GF} / \mathrm{F}$ Whatman, $47 \mathrm{~mm}$ diameter and $0.7 \mu \mathrm{m}$ pore size filter. A vacuum pressure pump and a filter holder were used to help in the filtration process. The filter was frozen and kept in the dark until further analysis. The residue on the filter was used to estimate the Chl- $a$ concentration in the laboratory. Extraction by acetone was the method adopted to estimate the Chl- $a$ concentration (Golterman, 1975).

Water samples were filtered in glass fiber filter GF/F Whatman ( $47 \mathrm{~mm}$ diameter and $0.7 \mu \mathrm{m}$ pore size) and stored frozen and in the dark to estimate TSS. The filters were dried in an oven at $105^{\circ} \mathrm{C}$ for $12 \mathrm{~h}$, desiccated and weighed to obtain the TSS. The filters were ignited at $550^{\circ} \mathrm{C}$ for $30 \mathrm{~min}$ in the muffle furnace, and then desiccated and weighed to acquire the ISS. Subtracting the ISS from the TSS yielded the OSS, and dividing each component of solids by filtered volume provided the concentrations of each constituent (APHA, 1998).

\subsection{Inherent optical properties (IOPs)}

$a_{p}(\lambda), a_{C D M}(\lambda)$ and $a_{\varphi}(\lambda)$ were estimated in laboratory and were used to assess the QAA performance. Water samples were collected from 20 sampling locations during both field trips (May 2014 and October 2014). $250 \mathrm{ml}$ of water from each location was filtered using GF/F Whatman glass fiber filters with $0.7 \mu \mathrm{m}$ pore size and $47 \mathrm{~mm}$ diameter, kept frozen and in the dark until the analysis to estimate $a_{p}(\lambda), a_{C D M}(\lambda)$ and $a_{\varphi}(\lambda)$. The measurements were acquired over $280-800 \mathrm{~nm}$ spectral range at $1 \mathrm{~nm}$ interval by using a 2600 UV-Vis spectrophotometer (Shimadzu, Kyoto, Japan) with dual beam and an integrating sphere. The optical density of the particulate materials was obtained from the first reading. After, the pigment in the filter was extracted with sodium chloride, the filter was measured again to determine the optical density of detritus. These optical densities were corrected for multiple scattering 
effects caused by the glass-fiber filter (Cleveland and Weidemann, 1993). The $a_{p}$ and $a_{d}$ were estimated from the corrected optical densities (Tassan and Ferrari, 1995, 1998, 2002). Finally, the $a_{\varphi}$ was obtained by subtracting the $a_{d}$ from $a_{p}$.

Water samples were filtered through a nylon membrane Whatman filter with $0.22 \mu \mathrm{m}$ pore size and $47 \mathrm{~mm}$ diameter to measure the CDOM (colored dissolved organic material) optical density $\left(A_{C D O M}\right)$. The filtrates were stored, kept cool and in the dark until the analysis. The samples were measured at room temperature using a quartz cuvette with $10 \mathrm{~cm}$ optical path. The measurements were acquired in a spectral range of $280-800 \mathrm{~nm}$ using a 2600 UV-Vis spectrophotometer (Shimadzu, Kyoto, Japan) with a single beam. Milli-Q water was used as blank reference. From the $A_{C D O M}$, CDOM absorption coefficients $\left(a_{\mathrm{CDOM}}\right)$ were calculated by using Eq. (3) (Bricaud et al., 1981).

$a_{\mathrm{CDOM}}=2.3 \frac{A_{\mathrm{CDOM}}(\lambda)}{l}$

where $A_{C D O M}(\lambda)$ is the optical density at wavelength $(\lambda)$ and $l$ is the cuvette path length in meters (Bricaud et al., 1981).

\subsection{Quasi-Analytical Algorithm (QAA)}

QAA is used to retrieve $a(\lambda)$ and $b_{b}(\lambda)$ coefficients from $R_{r s}$ and is based on the principle that $R_{r s}$ behavior depends on the IOPs of the ORCs present in the water (Lee et al., 2002). Table 5 shows the altered steps of the inversion model to derive $a(\lambda)$ and $b_{b}(\lambda)$. Several existing versions of the QAA were tested in this study, however, only five QAAs were further analyzed. Two criteria were used in selecting existing QAAs for further analysis, first, the QAA should be able to retrieve $a_{\varphi}$ at MERIS bands or at least at wavelengths typically associated with $a_{\varphi}$ and suitable for inland waters applications; and second, the QAA should not perform poorly, i.e., consistent under- or overestimation in retrieving $a_{\varphi}$ for this study site. Three out of the five were developed for open ocean and coastal waters: QAA_v4 (IOCCG, 2006), QAA_v5 (Lee et al., 2009), and QAA_v6 (Lee, 2014). QAA_v4 and QAA_v5 are recommended for water with low absorption coefficients, where, $R_{r s}(670)$ $<0.0065 \mathrm{sr}^{-1}$ (Lee et al., 2009; Lee, 2014). QAA versions developed for inland waters by Mishra et al. (2013, 2014) were also tested and were labeled as QAA_M13 and QAA_M14, respectively. Both QAA_M13 and QAA_M14 used $708 \mathrm{~nm}$ as the reference wavelength $\left(\lambda_{0}\right)$ and were parametrized to quantify PC concentration (Mishra et al., 2013, 2014). In turbid waters, the $\lambda_{0}$ should be shifted to longer wavelengths where water absorption is predominant in order to avoid strong interference from other ORCs (Lee et al., 2002, 2009; Mishra et al., 2014). Both QAA_M13 and QAA_M14 were parametrized and calibrated for waters with characteristics somewhat similar to BBHR. QAA_M13 and QAA_M14 were originally parametrized and calibrated using data collected at aquaculture ponds in Thad Cochran National Warmwater Aquaculture Center, Mississippi, USA. Ponds were used for catfish aquaculture and presented characteristics of high algal turbidity and primary productivity. A dataset comprising of 24 samples was used to calibrate QAA_M13, whereas, 20 samples were used to calibrate QAA_M14. Chl- $a$ concentration varied from $59.4 \mathrm{mg} \mathrm{m}^{-3}$ to $1376.6 \mathrm{mg} \mathrm{m}^{-3}$ and average of $293.3 \mathrm{mg} \mathrm{m}^{-3}$, while $a(443)$ range was of $4.99 \mathrm{~m}^{-1}$ to $47.21 \mathrm{~m}^{-1}$.

\subsubsection{Parameterization and calibration of the $Q A A$}

These different versions of QAA found in the literature frequently point to the fact that the IOPs for the water system must be taken into account in order to develop an accurate QAA. In inland waters, the bio-optical parameters depend on the geological and soil characteristics and runoff activities of the drainage basin. Therefore, it is expected that the versions found in the literature may not be suitable for reservoirs in tropical regions. Reparameterization and calibration of existing QAA may be the only option to improve their performances (Lee et al., 2009; Mishra et al., 2013, 2014; Lee, 2014). In this paper, empirical steps of the QAA were tuned based on the bio-optical characteristics of BBHR. Empirical steps were modified to improve the performance of the QAA and are indicated in Table 5. The version proposed in this paper retains the basic framework of QAA_v5 and is referred to as QAA_BBHR. $a(\lambda)$ and $b_{b}(\lambda)$ were retrieved from $r_{r s}$ measurements. The $r_{r s}$ can be analytically derived from $u$ (ratio of $b_{b}$ to the sum of $a$ and $b_{b}$ ) or empirically from $R_{r s}$ as shown in Eqs. (4) and (5).

$r_{r s}=g_{0} u(\lambda)+g_{1}[u(\lambda)]^{2}$

$r_{r s}=R_{r s} /\left(0.52+1.7 R_{r s}\right)$

where, $g_{0}=0.0895$ and $g_{1}=0.125$ are average of the values proposed by Gordon et al. (1988) and Lee et al. (1999) that vary with the phase function of particle and are not remotely measured (Lee et al., 2002). $u$ can be derived from $r_{r s}$ (Eq. (4)) as shown in Eq. (7).

$u=\frac{b_{b}}{a+b_{b}}$

$u=\frac{-g_{0}+\sqrt{\left(g_{0}\right)^{2}+4 g_{1} \times r_{r s}(\lambda)}}{2 g_{1}}$

Re-parameterization and calibration of QAA began by determining the $\lambda_{0}$. According to Lee et al. (2002), $\lambda_{0}$ is the position where the elastic scattering can be accurately measured and $a\left(\lambda_{0}\right)$ can be estimated from $r_{r s}\left(\lambda_{0}\right)$. In this study, $709 \mathrm{~nm}$ was selected as $\lambda_{0}$, assuming $a_{t}(709)$ equals to $a_{w}(709)$ (Lee et al., 2002, 2009; Mishra et al., 2014). At $709 \mathrm{~nm}$, the $a_{w}$ has a greater contribution toward the $a_{t}(\lambda)$. The average $a_{w}(709)$ contributed $75 \%$ toward the $a_{t}(709)$ in our dataset. That contribution was much higher in May (84.4\%) compared to October (65.5\%), whereas, the average $a_{w}(560)$ and $a_{w}(670)$ contributed $9.2 \%$ and $28.6 \%$ toward the corresponding $a_{t} \cdot a_{w}(\lambda)$ proposed by Pope and Fry (1997) and $b_{b w}(\lambda)$ proposed by Smith and Baker (1981) were adopted for the parameterization of QAA_BBHR.

Fig. 3 shows the average $a_{\varphi}$ and $a_{C D M}$ acquired from BBHR, in (a) May 2014 and (b) October 2014. It is noticeably clear that $a_{\varphi}(\lambda)$ and $a_{C D M}(\lambda)$ determine the shape and magnitude of $a_{t}(\lambda)$ at $555 \mathrm{~nm}$ and $665 \mathrm{~nm}$ as opposed to at $709 \mathrm{~nm}$. Contribution of $a_{\varphi}(\lambda)$ was $48.3 \%$ at $560 \mathrm{~nm}$ and $63.2 \%$ at $670 \mathrm{~nm}$, whereas, CDM absorption was $43.4 \%$ and $9.9 \%$ of $a_{t}$ at $560 \mathrm{~nm}$ and $670 \mathrm{~nm}$, respectively. It was also observed that $a_{C D M}(\lambda)$ has consistently higher influence than $a_{\varphi}(\lambda)$ toward $a_{t}(\lambda)$. Finally, the pigment absorption features were mainly, related to Chl- $a$ and PC.

$a\left(\lambda_{0}\right)$ is composed by the sum of $a_{w}\left(\lambda_{0}\right), a_{\varphi}\left(\lambda_{0}\right)$ and $a_{C D M}\left(\lambda_{0}\right)$ (Eq. (8)) and can be empirically derived from Eq. (9) (Lee et al., 2009).

$a\left(\lambda_{0}\right)=a_{w}\left(\lambda_{0}\right)+a_{\varphi}\left(\lambda_{0}\right)+a_{C D M}\left(\lambda_{0}\right)$

$a\left(\lambda_{0}\right)=a_{w}\left(\lambda_{0}\right)+10^{h 0+h 1 \chi+h 2 \chi^{2}}$

where $\chi$ is a value obtained from the ratio of $r_{r s}$ at different wavelengths associated with spectral features of pigments and CDOM (Eq. (10)) (Lee et al., 2009), $h_{0}, h_{1}$ and $h_{2}$ are the calibration coefficients of the polynomial fit between $a_{t-w}\left(\lambda_{0}\right)$ and $\chi$. Overall, $r_{r s}$ at wavelengths associated with absorption of pigments performs most accurately for the estimation of $a\left(\lambda_{0}\right)$.

$\chi=\log _{10}\left(\frac{r_{r s}\left(\lambda_{1}\right)+r_{r s}\left(\lambda_{2}\right)}{r_{r s}\left(\lambda_{0}\right)+r_{r s}\left(\lambda_{3}\right)^{2} \times r_{r s}\left(\lambda_{4}\right)^{-1}}\right)$ 

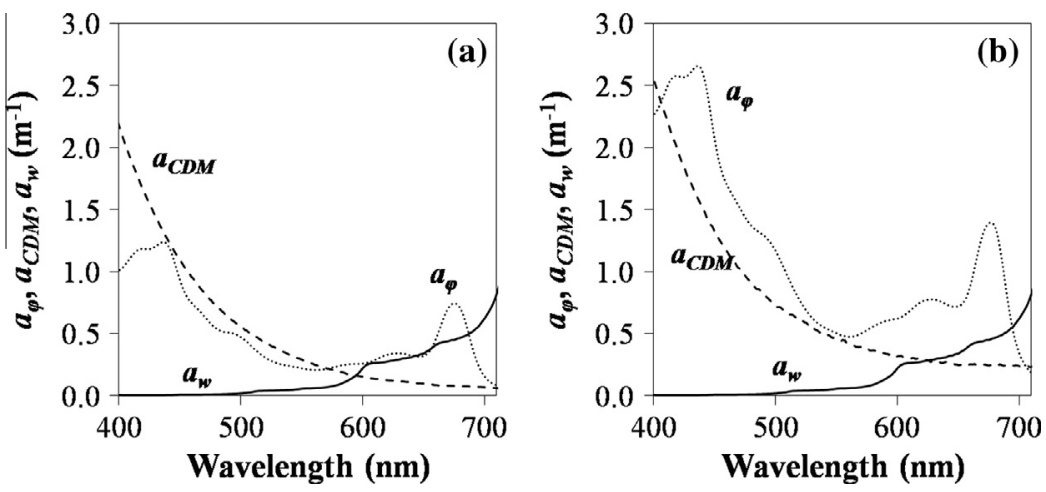

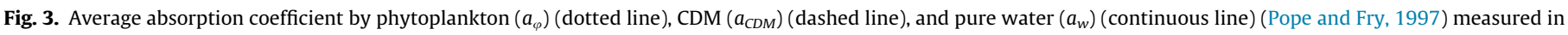
(a) May 2014 and (b) October 2014.

where $r_{r s}\left(\lambda_{0}\right)$ is below surface remote sensing reflectance at reference wavelength; $\lambda_{1}, \lambda_{2}, \lambda_{3}$, and $\lambda_{4}$ are wavelengths associated with dominant spectral features of the pigments and/or CDOM. Parameterization and calibration of $\chi$ was performed based on the band center of MERIS and Sentinel $2 \& 3$ sensors. The wavelengths $\left(\lambda_{1}\right.$, $\lambda_{2}, \lambda_{3}$ and $\lambda_{4}$ ) associated with the absorption by Chl- $a$ and PC $(443,620$, and $665 \mathrm{~nm})$ showed the best performance (Table 5). Different combinations were tested and the selection of the most adequate combination was based on NRSME and MAPE obtained in estimating $\mathrm{a}(\lambda)$ spectrum.

$b_{b p}\left(\lambda_{0}\right)$ was retrieved from $a\left(\lambda_{0}\right)$ analytically (Eq. (11)).

$b_{b p}\left(\lambda_{0}\right)=\frac{u\left(\lambda_{0}\right) a\left(\lambda_{0}\right)}{1-u\left(\lambda_{0}\right)}-b_{b w}\left(\lambda_{0}\right)$

where $u\left(\lambda_{0}\right)$ was calculated using Eq. (7) and $b_{b w}\left(\lambda_{0}\right)$ was adopted from Smith and Baker (1981).

$b_{b p}(\lambda)$ was derived from $b_{b p}\left(\lambda_{0}\right)$ based on Smith and Baker (1981).

$b_{b p}(\lambda)=b_{b}\left(\lambda_{0}\right)\left(\frac{\lambda_{0}}{\lambda}\right)^{\eta}$

Spectral power for $b_{b p}(\lambda)$ (Table 5) was also parametrized, changing the wavelength of denominator in Eq. (13). Based on calibration, the coefficients of $\eta$ were changed and evaluated to determine the most accurate coefficients. A value range of 1.0 to 1.9 was tested for $a, 1.3$ to 1.5 for $b$, and 0.1 to 0.8 for $c$. Although alterations were tested, the $\eta$ proposed by Lee et al. (2009) showed the best performance.

$\eta=a\left(b-c \times \exp \left(-d \frac{r_{r s}(443)}{r_{r s}(\lambda)}\right)\right)$

Spectral curve of $a(\lambda)$ was analytically estimated (Eq. (14)) from $u(\lambda), b_{b p}(\lambda)$ and $b_{b w}(\lambda)$.

$a(\lambda)=\frac{(1-u(\lambda))\left(b_{b w}(\lambda)+b_{b p}(\lambda)\right)}{u(\lambda)}$

$a_{\varphi}(\lambda)$ and $a_{C D M}(\lambda)$ were estimated from $a(\lambda) . \zeta$ and $\xi$ (Table 5) values depend on pigment composition, humic versus fulvic acids, and abundance of detritus. $\zeta$ is related to Chl- $a$ concentration or pigment absorption, while $\xi$ is related to the CDM absorption (Lee et al., 2002). $\zeta$ and $\xi$ were derived empirically based on Eqs. (15) and (16). Miscalibration of $\zeta$ and $\xi$ can lead to under- or overestimation of $a_{C D M}$ and $a_{\varphi}$.

$\zeta=\frac{a_{\varphi}(411)}{a_{\varphi}(443)}=a+\frac{b}{c+r_{r s}(443) / r_{r s}(560)}$

$\xi=\frac{a_{C D M}(411)}{a_{C D M}(443)}=e^{S(443-411)}$ $\xi$ was modified based on the $a_{C D M}$ spectral slope $(S)$ values obtained from laboratory analysis. Empirically, $S$ was also derived from the band ratio of absorption by pigments and CDOM (560 nm and $443 \mathrm{~nm}$, respectively) (Eq. (17)). A magnitude difference was detected between measured and estimated $S$, with average values of $0.161 \mathrm{~nm}^{-1}$ and $0.171 \mathrm{~nm}^{-1}$, respectively, and NRMSE (Normalized Root Mean Square Error) of $15.5 \%$. Thus, an alteration was applied to the intercept of the equation (Table 5) as presented in Eq. (17). Even after changing $S, a_{C D M}$ still produced negative values, consequently, $a_{\varphi}$ presented high overestimation, therefore, $\zeta$ (Table 5) also was calibrated. $\zeta$ works as multiplicative factor, always associated with a subtractive term. Therefore, $\zeta$ was likely being overestimated. Different values of offset (equation of Table 5) were tested, varying from 0.1 to 0.9 .

$S=a+\frac{b}{c+r_{r s}(443) / r_{r s}(560)}$

$a_{C D M}(443)$ was analytically derived (Eq. (18)) by removing the influence of $a_{\varphi}$ and $a_{w}$ from $a$ and by defining the proportion of CDM and phytoplankton absorption at wavelengths $411 \mathrm{~nm}$ and $443 \mathrm{~nm}$. $a_{C D M}$ is stronger at $411 \mathrm{~nm}$ than at $443 \mathrm{~nm}$ and phytoplankton absorbs more at $443 \mathrm{~nm}$ than at $411 \mathrm{~nm}$ (Carder et al., 1999). Therefore, the proportion of CDM and phytoplankton absorption can be determined using $\zeta$ and $\xi$ values. The spectral curve of $a_{C D M}(\lambda)$ can be analytically expressed by Eq. (19). $a_{\varphi}(\lambda)$ was derived from $a(\lambda)$ and $a_{C D M}(\lambda)$ using Eq. (20).

$a_{C D M}(443)=\frac{(a(411)-\zeta a(443))-\left(a_{w}(411)-\zeta a_{w}(411)\right)}{\xi-\zeta}$

$a_{C D M}(\lambda)=a_{C D M}(443) e^{-S(\lambda-443)}$

$a_{\varphi}(\lambda)=a_{t}(\lambda)-a_{w}(\lambda)-a_{C D M}(\lambda)$

\subsection{Application of derived IOPs to retrieve Chl-a concentration}

One of the interests in retrieving absorption coefficients of each ORC is to estimate their concentrations. Therefore, IOPs derived by QAA were used to parametrize bio-optical models for estimating Chl- $a$ which can be used as bio-indicator of phytoplankton biomass (Goodin et al., 1993). Phytoplankton absorbs strongly at $443 \mathrm{~nm}$ and commonly used ocean color algorithms utilize the bluegreen ratio to estimate Chl-a (O'Reilly et al., 1998; Carder et al., 1999). However, in eutrophic water bodies such as BBHR, absorption coefficients at blue region can be highly influenced by other components such as CDOM. Therefore, red-NIR wavelengths have been used in several bio-optical algorithms to estimate Chl- $a$ because these wavelengths have low interference from the absorption or backscattering coefficients of CDOM and detritus (Gitelson 
et al., 2008; Mishra and Mishra, 2012; Le et al., 2013; Matsushita et al., 2015). In highly turbid waters, $754 \mathrm{~nm}$ can also be used to minimize the effects from detritus and CDOM (Gitelson et al., 2008; Matsushita et al., 2015).

We used the $a_{\varphi}$ derived by QAA in redesigning the red-NR based Chl- $a$ algorithms to retrieve Chl- $a$ concentration. Comparison between the models was used as a way of evaluating the performance of the estimated $a_{\varphi}$. The two-band (2B), three-band (3B) indices (Gitelson et al., 2003) and normalized difference chlorophyll index (NDCI) (Mishra and Mishra, 2012) were tested. 2B and 3B indices were decomposed in terms of $a_{\varphi}$ and $a_{w}$ (semianalytical approaches) as proposed by Le et al. (2013). Similarly, an index based on absorption coefficients was proposed to replace NDCI. Wavelengths at 665, 709 and $754 \mathrm{~nm}$ (Gitelson et al., 2008; Mishra and Mishra, 2012) were used based on MERIS/Sentinel band configuration. Eqs. (21) and (22) show the structure of 2B index (Gitelson et al., 2008) using $a_{\varphi}$ and $a_{w}$ (Le et al., 2013). Similarly, Eqs. (23) and (24) show the 3B index (Gitelson et al., 2008) and Eqs. (25) and (26) show the architecture of NDCI (Mishra and Mishra, 2012). The models were calibrated by the least square method using a prediction interval with a confidence level of 0.95 .

$$
\begin{aligned}
& 2 B=R_{r s}(709) / R_{r s}(665) \\
& \psi_{1}=\left[a_{\varphi}(665)+a_{w}(665)\right] / a_{w}(709) \\
& 3 B=\left[R_{r s}^{-1}(665)-R_{r s}^{-1}(709)\right] \times R_{r s}(754) \\
& \psi_{2}=\left[a_{\varphi}(665)+a_{w}(665)-a_{\varphi}(709)-a_{w}(709)\right] / a_{w}(754) \\
& N D C I=\frac{R_{r s}(709)-R_{r s}(665)}{R_{r s}(709)+R_{r s}(665)} \\
& \psi_{3}=\frac{a_{w}(665)+a_{\varphi}(665)-a_{w}(709)}{a_{w}(665)+a_{C D M}(665)+a_{\varphi}(665)+a_{w}(709)+a_{C D M}(709)}
\end{aligned}
$$

\subsection{Validation}

Validation of the models was performed using the statistic metrics: root mean square error (RMSE) (Eq. (27)), normalized root mean square error (NRMSE) (Eq. (28)), mean absolute percentage error (MAPE) (Eq. (29)) and bias (Eq. (30)).

$$
R M S E=\left\{\frac{\sum_{i=1}^{n}\left[\left(x_{i}^{\text {estimated }}\right)-\left(x_{i}^{\text {measured }}\right)\right]^{2}}{n}\right\}^{1 / 2}
$$

$N R M S E=\frac{R M S E}{x_{\max }^{\text {measured }}-x_{\min }^{\text {measured }}} \times 100(\%)$

$M A P E=\frac{\sum_{i=1}^{n}\left(\left|\frac{x_{i}^{\text {estimated }}-x_{i}^{\text {measured }}}{x_{i}^{\text {measured }}}\right|\right)}{n}$

Bias $=\frac{1}{n} \sum_{i=1}^{n}\left(x_{i}^{\text {estimated }}-x_{i}^{\text {measured }}\right)$

\section{Results and discussion}

\subsection{Water quality parameters}

BBHR presents characteristics of highly productive waters with an average Chl- $a$ concentration of $120 \mathrm{mg} \mathrm{m}^{-3}$ in May and
$428.0 \mathrm{mg} \mathrm{m}^{-3}$ in October (Table 2). Elevated trophic levels occurred from May (Austral Autumn) to October (Austral Spring), 2014. The Chl- $a$ concentration showed a range of $17.7 \mathrm{mg} \mathrm{m}^{-3}$ to $279.9 \mathrm{mg} \mathrm{m}^{-3}$ in May, 2014 and a range of $263.2 \mathrm{mg} \mathrm{m}^{-3}$ to $797.8 \mathrm{mg} \mathrm{m}^{-3}$ in October, 2014. The minimum Chl- $a$ in October was close to the maximum Chl- $a$ concentration in May. An average OSS/TSS ratio of 0.83 in May and 0.9 in October was found, which explains the dominance of organic matter in the total particulate material in BBHR. $a_{\varphi}(\lambda)$ values were much higher than $a_{C D M}(\lambda)$ from May to October, corroborating that turbidity and TSS were mainly associated with phytoplankton biomass in BBHR. There was a considerable increase in the average $a_{\varphi}(440)$ from May to October, with a range of $1.25 \mathrm{~m}^{-1}$ to $2.81 \mathrm{~m}^{-1}$, whereas, average $a_{C D M}(412)$ ranged from $1.86 \mathrm{~m}^{-1}$ to $2.12 \mathrm{~m}^{-1}$. In other words, average $a_{\varphi}(440)$ increased $55.5 \%$ and $a_{C D M}(412)$ increased only $12.3 \%$. Average $a_{\varphi}(\lambda)$ and $a_{C D M}(\lambda)$ (Fig. 3) and $a_{\varphi}(440) / a_{C D M}(440)$ (Table 2) show that the contribution of $a_{C D M}(\lambda)$ to the $a(\lambda)$ was higher than $a_{\varphi}(\lambda)$, in May, in contrast to October when the contribution of $a_{\varphi}(\lambda)$ was higher. The elevated levels of Chl- $a$ led to rising algal turbidity with an average of 5.2 NTU in May and 18.6 NTU in October as a result the Secchi disk depth decreased significantly with maximum value of $2.3 \mathrm{~m}$ in May and a maximum of $0.8 \mathrm{~m}$ in October. On the other hand, the dataset collected in September 2015 exhibited very similar values when compared with May dataset (Table 2).

\section{2. $a(\lambda)$ retrieval}

Table 5 shows the parameterizations and calibrations accomplished in QAA_BBHR. Among the four alterations carried out, two of them are very important for accurate estimation of $a_{t}(\lambda)$. First, the selection of the wavelength suitable to estimate the absorption coefficient of the pigments, detritus and CDOM made a difference as well as the calibration of $\chi$. Fig. 4 shows the $a_{t}(\lambda)$ spectra determined by the spectrophotometer (Fig. 4a), QAA_v4 (Fig. 4b) (Lee et al., 2009), QAA_v5 (Fig. 4c) (Lee et al., 2009), QAA_v6 (Fig.4d) (Lee, 2014), QAA_M13 (Fig. 4e) (Mishra et al., 2013), QAA_M14 (Fig.4f) (Mishra et al., 2014) and QAA_BBHR (Fig. 4g). Among all the versions of QAA tested, based on NRMSE, MAPE and bias parameters, the most recent version of the QAA proposed by Mishra et al. (2014) presented the best performance in estimating $a_{t}(\lambda)$, because this version was fitted for waters with bio-optical status somewhat similar to BBHR. Meanwhile, QAA_v4, QAA_v5 and QAA_v6 underestimated $a_{t}(\lambda)$, since they were developed for waters with very low $a_{t}(\lambda)$. Although QAA_M13 is similar to QAA_M14, it overestimated $a_{t}(\lambda)$ most likely due to the parametrization of $\chi$ which has been fitted for more productive waters than BBHR. As expected, the version fitted with BBHR dataset (QAA_BBHR) showed the best performance, because it is considered the bio-optical status of the reservoir itself.

Parameterization and calibration of $\chi$ presented considerable change in $a_{t}(\lambda)$ estimation, even though $\chi$ is defined as importance of second order (Lee et al., 2002). The QAA versions parametrized for oceanic and coastal waters highly underestimated $a_{t}(\lambda)$ across all wavelengths. QAA_v4, QAA_v5 and QAA_v6 showed an average bias ranging from $-1.31 \mathrm{~m}^{-1}$ to $-1.04 \mathrm{~m}^{-1}$. The most underestimation was observed at $412 \mathrm{~nm}$ (range of $-3.18 \mathrm{~m}^{-1}$ for QAA_v4 to $-2.79 \mathrm{~m}^{-1}$ for QAA_v5), and the least was observed at $560 \mathrm{~nm}$ (range of $-0.5 \mathrm{~m}^{-1}$ for QAA_v4 to $-0.36 \mathrm{~m}^{-1}$ for QAA_v5). The least accurate performance to retrieve $a_{t}(\lambda)$ was exhibited by QAA_v4, due to parameters used to determine $\chi$. The main difference between QAA_v4 and QAA_v5 is the wavelength used, $640 \mathrm{~nm}$ in QAA_v4 and $667 \mathrm{~nm}$ in QAA_v5. Taking into account the maximum Chl- $a$ absorption in red light is at $675 \mathrm{~nm}, 667 \mathrm{~nm}$ is more representative that pigment than $640 \mathrm{~nm}$. Both versions used $555 \mathrm{~nm}$ as $\lambda_{0}$ to estimate $\chi$ (Lee et al., 2009), which has been shown as inappropriate for inland waters (Le et al., 2009; Mishra et al., 
Table 2

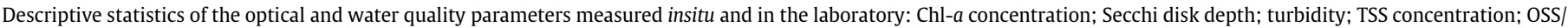

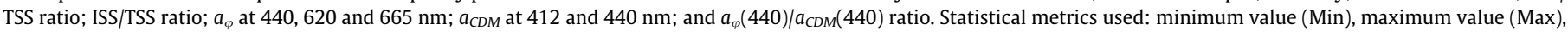
mean, median, standard deviation (SD) and coefficient of variation (CV) in percentage (\%), that is $\mathrm{CV}=(\mathrm{SD} / \mathrm{mean}) * 100$.

\begin{tabular}{|c|c|c|c|c|c|c|}
\hline & Min & Max & Mean & Median & SD & $\mathrm{CV}(\%)$ \\
\hline \multicolumn{7}{|c|}{ May 5-9, 2014 dataset, $n=18$ stations } \\
\hline Chl- $a, \mathrm{mg} \mathrm{m}^{-3}$ & 17.7 & 279.9 & 120.4 & 101.3 & 70.3 & 58.4 \\
\hline Secchi disk depth, m & 0.8 & 2.3 & 1.5 & 1.4 & 0.4 & 26.7 \\
\hline Turbidity, NTU & 1.7 & 12.5 & 5.2 & 5 & 2.4 & 46.2 \\
\hline TSS, $\mathrm{g} \mathrm{m}^{-3}$ & 3.6 & 16.3 & 7.2 & 6.5 & 3.3 & 45.8 \\
\hline OSS/TSS & 0.45 & 0.98 & 0.83 & 0.87 & 0.12 & 14.5 \\
\hline ISS/TSS & 0.02 & 0.55 & 0.17 & 0.13 & 0.12 & 7.1 \\
\hline$a_{\varphi}(440), \mathrm{m}^{-1}$ & 0.31 & 2.62 & 1.25 & 1.05 & 0.63 & 50 \\
\hline$a_{\varphi}(620), \mathrm{m}^{-1}$ & 0.12 & 0.78 & 0.34 & 0.31 & 0.18 & 51.9 \\
\hline$a_{\varphi}(665), \mathrm{m}^{-1}$ & 0.2 & 1.26 & 0.61 & 0.52 & 0.28 & 45.1 \\
\hline$a_{C D M}(412), \mathrm{m}^{-1}$ & 1.57 & 2.68 & 1.86 & 1.78 & 0.27 & 14.5 \\
\hline$a_{C D M}(440), \mathrm{m}^{-1}$ & 1.06 & 1.91 & 1.26 & 1.21 & 0.21 & 16.5 \\
\hline$a_{\varphi}(440) / a_{C D M}(440)$ & 0.3 & 2.15 & 0.99 & 0.84 & 0.49 & 49.8 \\
\hline \multicolumn{7}{|c|}{ October $13-16,2014, n=20$ stations } \\
\hline Chl- $a, \mathrm{mg} \mathrm{m}^{-3}$ & 263.2 & 797.8 & 428.7 & 368.9 & 154.5 & 36 \\
\hline Secchi disk depth, m & 0.4 & 0.8 & 0.6 & 0.6 & 0.1 & 16.7 \\
\hline Turbidity, NTU & 11.6 & 33.2 & 18.6 & 17.6 & 5.3 & 28.5 \\
\hline TSS, $\mathrm{g} \mathrm{m}^{-3}$ & 10.8 & 44 & 22 & 21.2 & 7 & 31.8 \\
\hline OSS/TSS & 0.8 & 0.96 & 0.9 & 0.9 & 0.1 & 11.1 \\
\hline ISS/TSS & 0.04 & 0.2 & 0.1 & 0.1 & 0.05 & 40 \\
\hline$a_{\varphi}(440), \mathrm{m}^{-1}$ & 1.56 & 5.59 & 2.81 & 2.57 & 1.03 & 36.8 \\
\hline$a_{\varphi}(620), \mathrm{m}^{-1}$ & 0.44 & 1.56 & 0.79 & 0.76 & 0.31 & 39.4 \\
\hline$a_{\varphi}(665), \mathrm{m}^{-1}$ & 0.63 & 2.1 & 1.16 & 1.08 & 0.43 & 36.8 \\
\hline$a_{C D M}(412), \mathrm{m}^{-1}$ & 1.6 & 3.26 & 2.12 & 2.11 & 0.36 & 16.8 \\
\hline$a_{C D M}(440), \mathrm{m}^{-1}$ & 1.02 & 2.6 & 1.45 & 1.42 & 0.33 & 22.6 \\
\hline$a_{\varphi}(440) / a_{C D M}(440)$ & 0.64 & 2.61 & 1.36 & 1.2 & 0.56 & 48.9 \\
\hline \multicolumn{7}{|c|}{ September $13-15,2015, n=24$ stations } \\
\hline Chl- $a, \mathrm{mg} \mathrm{m}^{-3}$ & 62.8 & 245.7 & 127.1 & 106.5 & 51.3 & 40.4 \\
\hline Secchi disk depth, m & 1 & 1.6 & 1.3 & 1.2 & 0.2 & 16.6 \\
\hline Turbidity, NTU & 3.1 & 6.8 & 4.2 & 4.1 & 0.8 & 20.3 \\
\hline TSS, $\mathrm{g} \mathrm{m}^{-3}$ & 1.6 & 8.4 & 5.6 & 5.8 & 1.8 & 32 \\
\hline
\end{tabular}

$2013,2014)$. The other wavelengths used to parametrize $\chi$ were absorption bands of Chl- $a$ at $443 \mathrm{~nm}$ and $490 \mathrm{~nm}$ (Lee et al., 2009). On the other hand, QAA_v6 do not use the $\chi$ factor to estimate the absorption coefficients of ORCs $\left[a_{\varphi}(\lambda)\right.$ and $\left.a_{C D M}(\lambda)\right]$. To estimate $a_{t}\left(\lambda_{0}\right)$, QAA_v6 considered a power function $\left(y=x^{n}\right)$ using $R_{r s}$ at wavelengths 443,490 and $670 \mathrm{~nm}$ to represent the sum of $a_{\varphi}\left(\lambda_{0}\right)$ and $a_{C D M}\left(\lambda_{0}\right)$ (Lee, 2014). QAA_v6 was proposed for situations where $R_{r s}(670)>0.0065 \mathrm{sr}^{-1}$ (Lee, 2014) and showed lower accuracy than QAA_v5 in BBHR, where the overall average $R_{r s}(670)$ was of $0.0059 \mathrm{sr}^{-1}$ (average of $0.004 \mathrm{sr}^{-1}$ in May and $0.0076 \mathrm{sr}^{-1}$ in October). The use of $670 \mathrm{~nm}$ as $\lambda_{0}$ may have impaired its performance in inland waters, since this wavelength is highly influenced by the ORCs absorption.

The models proposed for inland water such as QAA_M13 and QAA_M14 were parametrized and calibrated in highly turbid productive waters (Mishra et al., 2013, 2014). In such waters, there is a high influence of $a_{\varphi}\left(\lambda_{0}\right)$ and $a_{C D M}\left(\lambda_{0}\right)$ over $a_{t}\left(\lambda_{0}\right)$ at $555 \mathrm{~nm}$ and $667 \mathrm{~nm}$. Thus, $555 \mathrm{~nm}$ and $667 \mathrm{~nm}$ are not suitable as $\lambda_{0}$ in those waters (Le et al., 2009; Mishra et al., 2013, 2014; Li et al., 2013, 2015). For that reason, $708.75 \mathrm{~nm}$ (red edge region and central wavelength of band 9 of the MERIS sensor) has been used as $\lambda_{0}$; where $a_{w}\left(\lambda_{0}\right)$ is expected to dominate $a_{t}\left(\lambda_{0}\right)$ (Lee et al., 2002; Mishra et al., 2014, 2013). Other researchers also tuned QAA using wavelength around $708.75 \mathrm{~nm}$ as $\lambda_{0}$. For example, Le et al. (2009) conducted a study in Taihu Lake, China where they used $710 \mathrm{~nm}$ as $\lambda_{0}$. The shifting of $\lambda_{0}$ to red-edge region produced a major improvement in $a_{t}\left(\lambda_{0}\right)$ estimation accuracy. In their research (Li et al., 2013, 2015), a $\lambda_{0}$ was not adopted at every step. Nevertheless, the estimation of $a_{t-w}(\lambda)$ was performed using $R_{r s}(709)$ and $b_{b}(709) .753 \mathrm{~nm}$ was also used as $\lambda_{0}$ in three turbid Asian lakes, and $\lambda_{0}$ was shifted to prevent the interference of the high turbidity on $a_{t}\left(\lambda_{0}\right)$ (Yang et al., 2013). This modification produced good results in $a(\lambda)$ estimation for BBHR, similar to QAA_M2014, show- ing that longer wavelengths are more appropriate for eutrophic inland waters.

QAA_M13 and QAA_M14 overestimated $a_{t}(\lambda)$ in BBHR (Fig. 4). Although both models were originally developed to quantify PC in hyper-eutrophic waters $\left(\lambda_{1}=443 \mathrm{~nm}, \lambda_{2}=620 \mathrm{~nm}, \lambda_{3}=620 \mathrm{~nm}\right.$ and $\lambda_{4}=443 \mathrm{~nm}$ ) (Mishra et al., 2013, 2014) and share the same wavelengths to parametrize $\chi$, QAA_M13 showed higher overestimation than QAA_M14. QAA_M13 yielded an average bias of $0.65 \mathrm{~m}^{-1}$ with minimum and maximum being $0.21 \mathrm{~m}^{-1}(709 \mathrm{~nm})$ and $1.1 \mathrm{~m}^{-1}(620 \mathrm{~nm})$, whereas, QAA_M14 yielded an average bias of $0.41 \mathrm{~m}^{-1}$, with minimum and maximum of $0.12 \mathrm{~m}^{-1}(709 \mathrm{~nm})$ and $0.88 \mathrm{~m}^{-1}(620 \mathrm{~nm})$. The suitable calibration and parameterization of $\chi$ played an important role, changing significantly the estimation of $a_{t}(\lambda)$. Even though samples used to calibrate QAA_M13 and QAA_M14 were collected in the same environment, they produced different results for each dataset, due to fits carried out in the empirical steps (Mishra et al., 2013, 2014).

Different wavelength combinations were tested to parametrize $\chi$ with the final aim of estimating Chl- $a$ concentration. The best combinations of bands were $709 \mathrm{~nm}$ as $\lambda_{0}$ and the wavelengths representing absorption by Chl- $a$ and PC such as $\lambda_{1}=443 \mathrm{~nm}$, $\lambda_{2}=665 \mathrm{~nm}, \lambda_{3}=620 \mathrm{~nm}$ and $\lambda_{4}=443 \mathrm{~nm}$. Parameterization and calibration coefficients are presented in Table 5. Although $620 \mathrm{~nm}$ is associated with the absorption by PC and the band combination using only 443, 665 (Weaver and Wrigley, 1994; Mishra et al., 2009) and $709 \mathrm{~nm}$ presented good results, $620 \mathrm{~nm}$ was kept in $\chi$ calibration. Inclusion of $620 \mathrm{~nm}$ improved the estimation as shown in Fig. 5. This improvement can be associated with the predominance of cyanobacteria in BBHR. The absorption by PC pigment is clearly observed in $R_{r s}$ spectra at $620 \mathrm{~nm}$ (Fig. 2). Both Chl- $a$ and PC are present in cyanobacteria, consequently, these pigments can be highly correlated in environments where cyanobacteria is the predominant species (Simis et al., 2005; Mishra et al., 

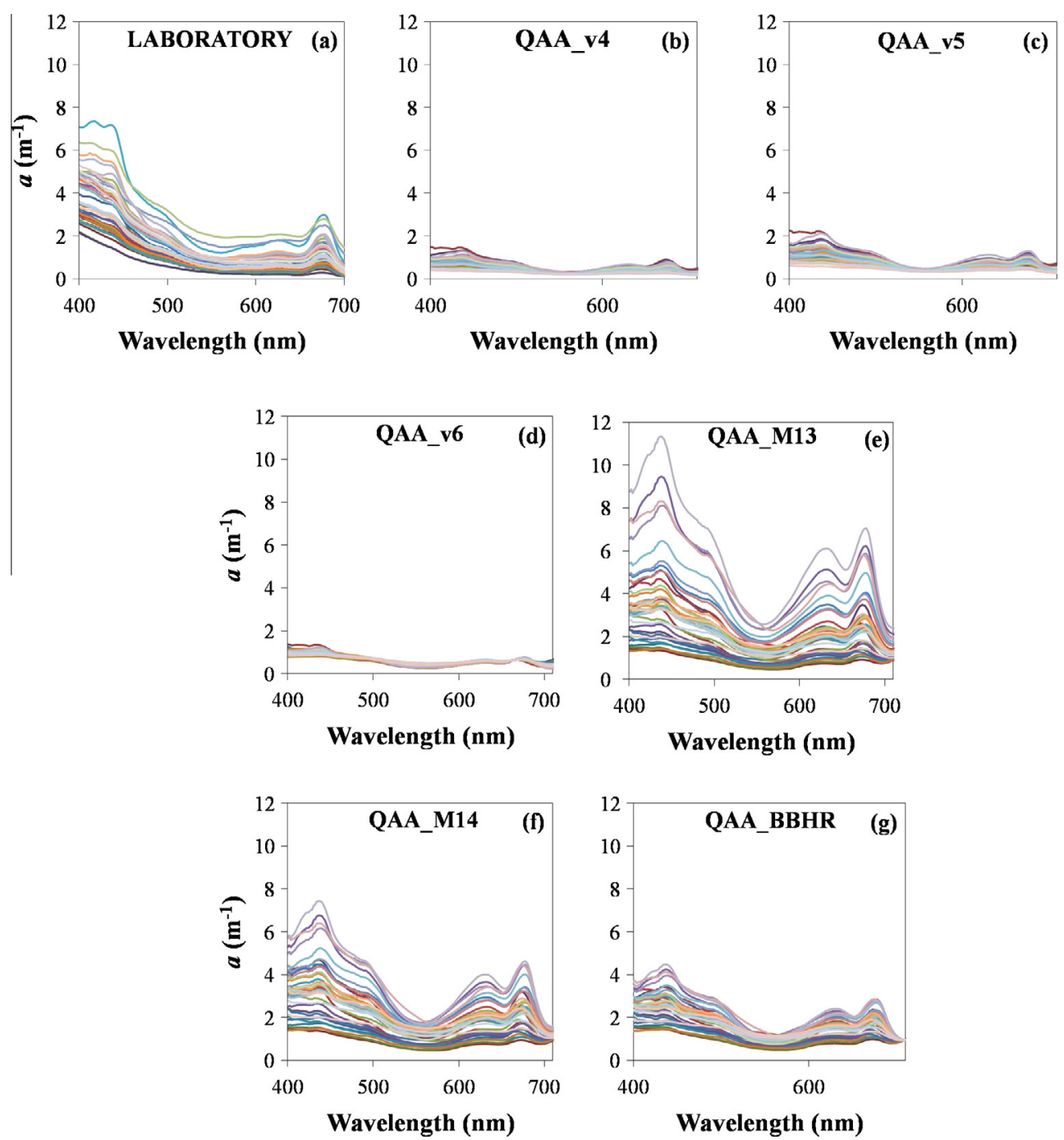

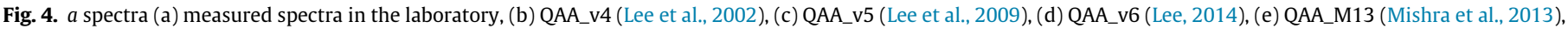
(f) QAA_M14 (Mishra et al., 2014) and (g) QAA_BBHR.

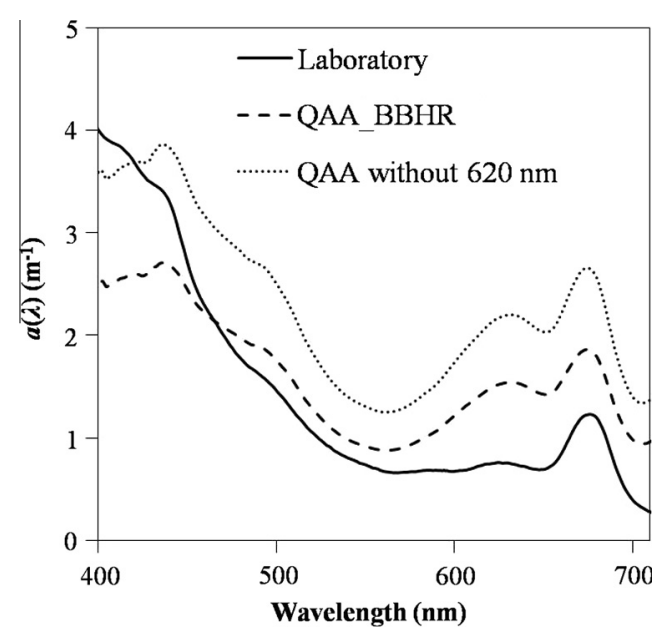

Fig. 5. Comparison of the total absorption coefficients measured in laboratory and obtained using QAA_BBHR and QAA parametrized without $620 \mathrm{~nm}$.

2009, 2014). Parameterization and calibration of QAA_BBHR (Fig. $4 \mathrm{~g}$ ) considerably improved the estimation of $a_{t}(\lambda)$. Comparing the $a_{t}(\lambda)$ spectra between laboratory measurements and
QAA_BBHR showed a clear underestimation at shorter wavelengths with a maximum bias of $-1.42 \mathrm{~m}^{-1}$ at $412 \mathrm{~nm}$. Additionally, there was a slight overestimation at $681 \mathrm{~nm}$, with a bias of $0.06 \mathrm{~m}^{-1}$. Overall, the model showed an average bias of $-0.11 \mathrm{~m}^{-1}$.

Fig. 6 shows the relationship between the measured versus estimated $a$ at 560, 665 and $705 \mathrm{~nm}$, wavelengths commonly used in estimating TSS concentration in inland waters. Despite of that, the analysis was conducted considering nine MERIS/Sentinel$2 \mathrm{~A} / 3 \mathrm{~A}$ central wavelengths $(412,443,510,560,620,665,681$ and $709 \mathrm{~nm})$. QAA_v4 and QAA_v5, proposed for sites where $R_{r s}(670)<0.0065 \mathrm{sr}^{-1}$ (Lee, 2014), produced maximum $R^{2}$ at $709 \mathrm{~nm}$ ( 0.21 and 0.19 , respectively) and minimum $R^{2}$ at $443 \mathrm{~nm}$ (0.0001 and 0.0037, respectively). QAA_v6 presented the maximum $R^{2}$ of 0.33 at $681 \mathrm{~nm}$ associated with Chl- $a$ fluorescence (Gordon, 1979), and inexpressible $R^{2}$ (of $2 \times 10^{-16}$ ) at $665 \mathrm{~nm}$. Both QAA_M13 and QAA_M14 presented the maximum $R^{2}$ at $681 \mathrm{~nm}$ (0.43 and 0.51 , respectively) and minimum $R^{2}$ at $709 \mathrm{~nm}(0.17$ and 0.24 , respectively). QAA_BBHR indicated slight improvement in terms of $R^{2}$ with maximum of 0.55 at $681 \mathrm{~nm}$. Surprisingly, $709 \mathrm{~nm}$ showed the worst fit with an inexpressible $R^{2}$.

QAA_BBHR primarily overestimated at shorter wavelengths. Three points 3, 7, and 19 (P3, P7 and P19, respectively) collected in October 2014 (Fig. 2a) fell farthest from the 1:1 line at 412 and $443 \mathrm{~nm}$ (Fig. 6f). The P3 is located in the largest region of the 

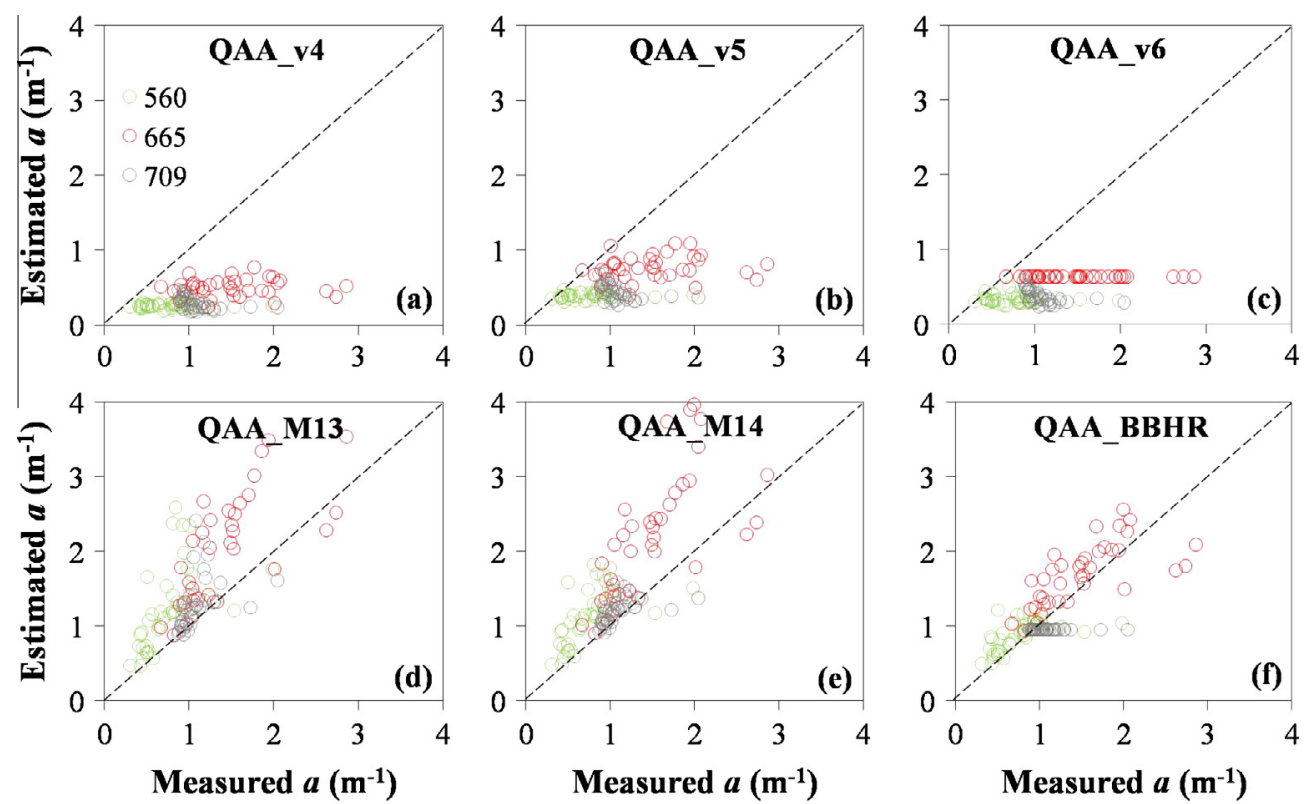

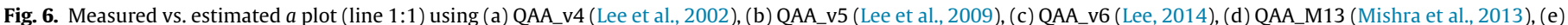
QAA_M14 (Mishra et al., 2014) and (f) QAA_BBHR.

reservoir before the channel narrows (see Fig. 1 for location), which produces algal blooms, mainly during austral summer. This point exhibited the highest $a_{t}(\lambda)$ in October, associated with the maximum $a_{\varphi}(\lambda)$ found in BBHR. According to laboratory measures, P7 presented the second highest $a_{t}(\lambda)$; however, in this case related to the greatest absorption by CDOM. On the contrary, P19 located in Tietê River before its confluence with Piracicaba River presented a unique $a_{t}(\lambda)$ spectrum, with strong absorption at blue and red regions, but intermediate absorption from 560 to $630 \mathrm{~nm}$. Different $a_{t}(\lambda)$ at P19 is a combination of high $a_{C D M}(\lambda)$ and $a_{\varphi}(\lambda)$. In addition, P19 exhibited a greater absorption at $460 \mathrm{~nm}$, which is not observed at other locations.

Fig. 7 shows the NRMSE and MAPE produced by all models in estimating $a_{t}(\lambda)$. QAA_v4, QAA_v5 and QAA_v6 presented similar NRMSE and MAPE values, being that the highest average errors were yielded by QAA_v4. 412 and $709 \mathrm{~nm}$ exhibited the highest errors, while intermediate wavelengths presented the lowest errors, with minimum at $560 \mathrm{~nm}$. QAA_v5 presented the lowest NRMSE and MAPE at $620 \mathrm{~nm}$ (PC absorption feature) among all models (NRMSE of $30.84 \%$ and MAPE of $32.63 \%$ ). QAA_v5 presented better performance than QAA_v6, due to the selection of $\lambda_{0}$ at $670 \mathrm{~nm}$, because it is highly influenced by ORCs absorption, mainly Chl- $a$, and one of the criterion for selection of $\lambda_{0}$ is that the influence of ORCs absorption should be minimum.

Among all five published QAA, QAA_M14 proposed by Mishra et al. (2014) presented the best performance to estimate $a_{t}(\lambda)$, with the lowest average NRMSE of $33.81 \%$ and MAPE of 46.55\%. Errors were the lowest at $709 \mathrm{~nm}$, with NRMSE of $20.19 \%$ and MAPE of 16.47\%. Prediction at the PC absorption band was highly erroneous with a NRMSE of $56.54 \%$ and MAPE of $88.9 \%$. Although QAA_M13 was parametrized with the same data as QAA_M14 from turbid productive waters, this version did not produce the same satisfactory results. QAA_M13 presented similar errors as QAA_v5, with an average NRMSE of $49.58 \%$ and MAPE of $56.6 \%$; however, QAA_v5 underestimated $a_{t}(\lambda)$, whereas, QAA_M13 overestimated the variable. QAA_turbid proposed by Yang et al. (2013) exhibited intermediate results between QAA_M14 and QAA_M13, with average NRMSE of $37.32 \%$ and MAPE of 59.37\%. However, QAA_turbid was not included for comparison mainly because of consistency purposes. All QAAs compared in this study predict IOPs including $a_{\varphi}$ at MERIS wavelengths except QAA_turbid which retrieves $a_{\varphi}$ at just one wavelength, at $443 \mathrm{~nm}$, where there is a strong influence of $a_{C D O M}$, and a wavelength not commonly used for $a_{\varphi}$ estimation in inland waters. The difference between QAA_M13 and QAA_M14 is basically the calibration parameters $h_{0}, h_{1}$ and $h_{2}$ (Eq. (9)), showing the importance of a suitable calibration during this step. Overall, QAA_M13 performed better at shorter wavelengths (NRMSE: $28.7 \%$ and MAPE: $27.1 \%$ at $412 \mathrm{~nm}$ ) and the poorest results were obtained at $620 \mathrm{~nm}$ (NMRSE: 78.95\% and MAPE: 103.93\%). QAA_M13 (Mishra et al., 2013) and QAA_M14 (Mishra et al., 2014) were originally designed to quantify PC; however, surprisingly, these versions produced high errors at $620 \mathrm{~nm}$ in BBHR. A possible reason for the overestimation of absorption by pigments could be the package effect. The package effect or pigment packaging flats $a_{\varphi}(\lambda)$ spectrum with the increase of phytoplankton biomass (Roesler et al., 1989; Bricaud et al., 1995; Carder et al., 1999; Ciotti et al., 2002). This effect is clearly observed in laboratorial measurements where samples with high Chl- $a$ concentration exhibited low $a_{\varphi}(\lambda)$. Therefore, QAA did not predict the flattening in $a_{t}(\lambda)$, showing the increase the pigment absorption features proportionally with the elevation of phytoplankton biomass. The above analysis reiterates the fact that calibration of $\chi$ plays a crucial role to retrieve $a_{t}(\lambda)$.

The combinations of wavelengths to parametrize $\chi$ and the calibration parameters obtained to derive $a_{t-w}\left(\lambda_{0}\right)$, and consequently $a_{t}\left(\lambda_{0}\right)$ was essential for the accurate estimation of $a_{t}(\lambda)$. The new parametrization yielded an average NRMSE of $21.88 \%$ and MAPE of $28.27 \%$, with best performance at $681 \mathrm{~nm}$, the wavelength associated with Chl- $a$ fluorescence (Gordon, 1979), with a NRMSE of $16.53 \%$ and a MAPE of $17.43 \%$. On the other hand, the algorithm exhibited its poorest performance at $620 \mathrm{~nm}$ (NRMSE: $37.29 \%$ and MAPE: 117.78\%) as QAA_M13 and QAA_M14, likely associated with pigment packaging (Roesler et al., 1989; Bricaud et al., 1995).

\section{3. $a_{C D M}(\lambda)$ retrieval}

Fig. 8 shows the $a_{C D M}(\lambda)$ spectra estimated by each QAA version tested in this study. None of the existing QAA versions yielded satisfactory results for $a_{C D M}(\lambda)$ in BBHR. It indicated the necessity of accurately parameterizing and calibrating the steps leading to 

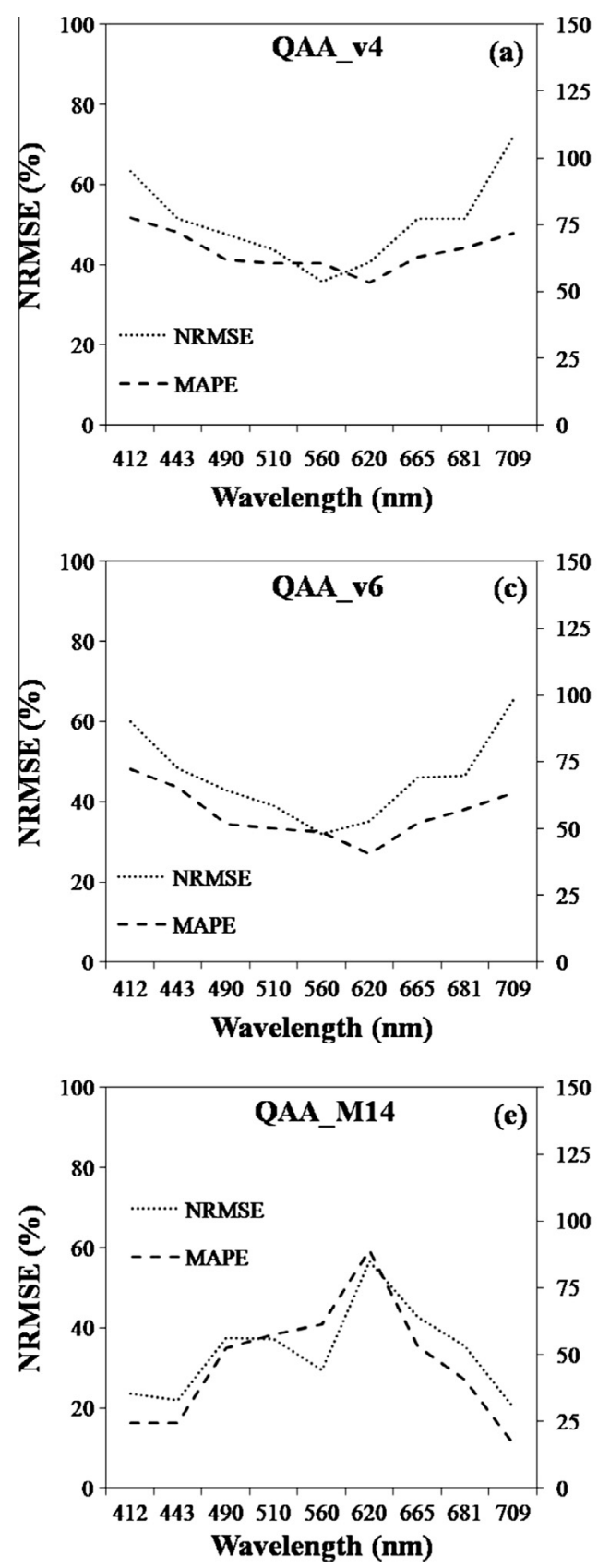
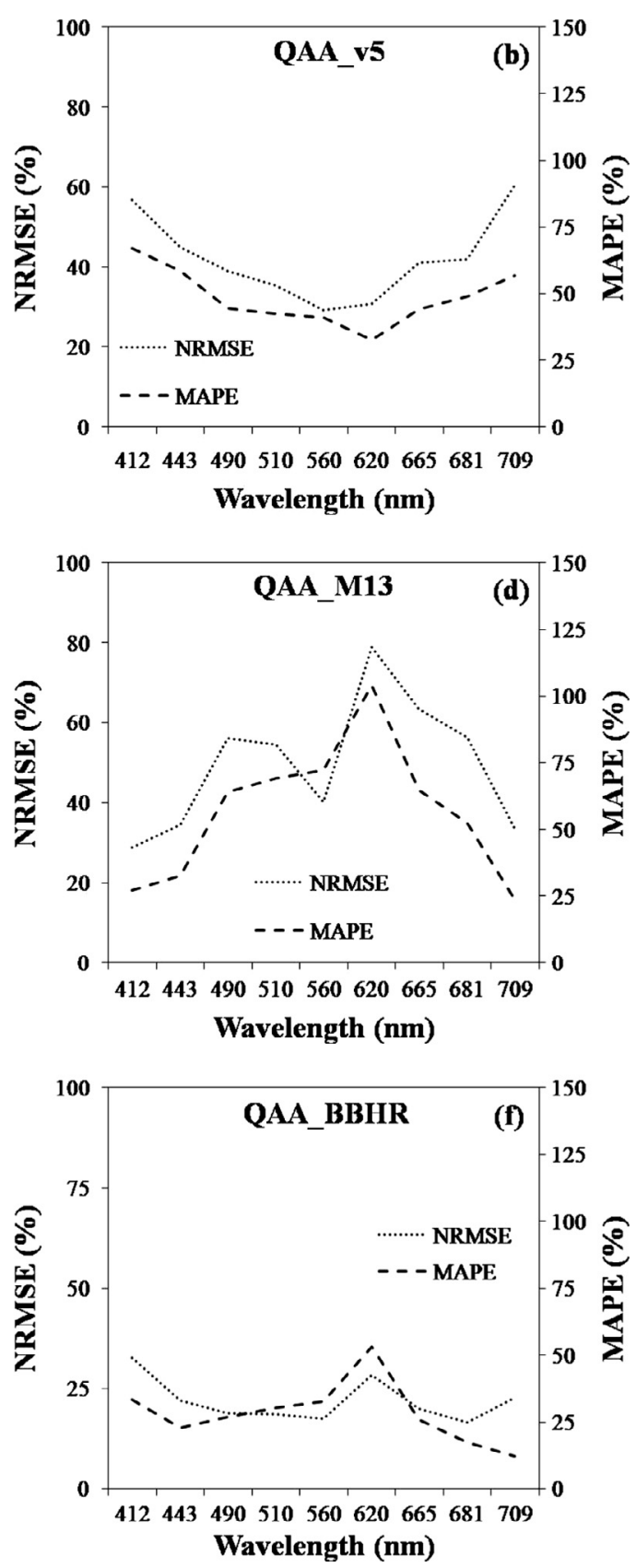

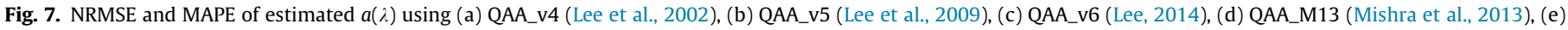
QAA_M14 (Mishra et al., 2014) and (f) QAA_BBHR.

$a_{C D M}(\lambda)$ estimation in the QAA. Accurate retrieval of $a_{C D M}(\lambda)$ and $a_{\varphi}(\lambda)$ depends basically on the accurate estimation of $a_{t}(\lambda)$ (Lee et al., 2010; Mishra et al., 2014), as well as suitable parameterization and calibration of $\xi$ and $\zeta$.

QAA_v5, QAA_v6, QAA_M13, and QAA_M14 versions yielded negative values for samples P4, P5, P10, P11 and P16 at shorter wavelengths. With the exception of P5, the negative values were computed for samples collected during the second field campaign (October 2014) when the $a_{C D M}(\lambda)$ were higher. Lee et al. (2010) and Mishra et al. (2014) have discussed obtaining negative values for $a_{C D M}(\lambda)$ in their QAA. Mishra et al. (2014) verified that negative $a_{C D M}(\lambda)$ did not impair the retrieval of $a_{\varphi}(\lambda)$ using red and NIR bands; however, the use of $a_{C D M}(\lambda)$ derived by QAA is preventable. QAA_v5, QAA_v6, QAA_M13 and QAA_M14 practically adopted the same model to estimate $\zeta$ and $\xi$ (Eqs. (15) and (16), respectively) unlike QAA_v4. Although the models adopted by QAA_v4 to estimate $\zeta$ and $\xi$ have not yielded negative values, they still were not suitable to estimate $a_{C D M}(\lambda)$ in BBHR, showing a high underestimation, with a negative bias of $-0.53 \mathrm{~m}^{-1}$. Thus, the underestimation of $a_{C D M}(\lambda)$ cannot only be associated with the underestimation associated with $a_{t}(\lambda)$, since QAA_M13 and QAA_M14 have overestimated $a_{t}(\lambda)$ and also yielded negative values. According to Mishra et al. (2014), overestimation of $\xi$ and underestimation of $\zeta$ can also underestimate $a_{C D M}(443)$. Therefore, QAA_BBHR was calibrated to fit $\zeta$ and $\xi$ and solve the problem of underestimation of $a_{C D M}(\lambda)$ and, consequently, overestimation of $a_{\varphi}(\lambda)$.

The slight overestimation of $S$ was the first problem detected. The existing versions tested in this study use models that produce $S$ value around $0.015 \mathrm{~nm}^{-1}$. QAA_v4 uses a fixed value of $S$ equals to 0.015 to estimate $\xi$. Although QAA_v5, QAA_v6, QAA_M13 and 

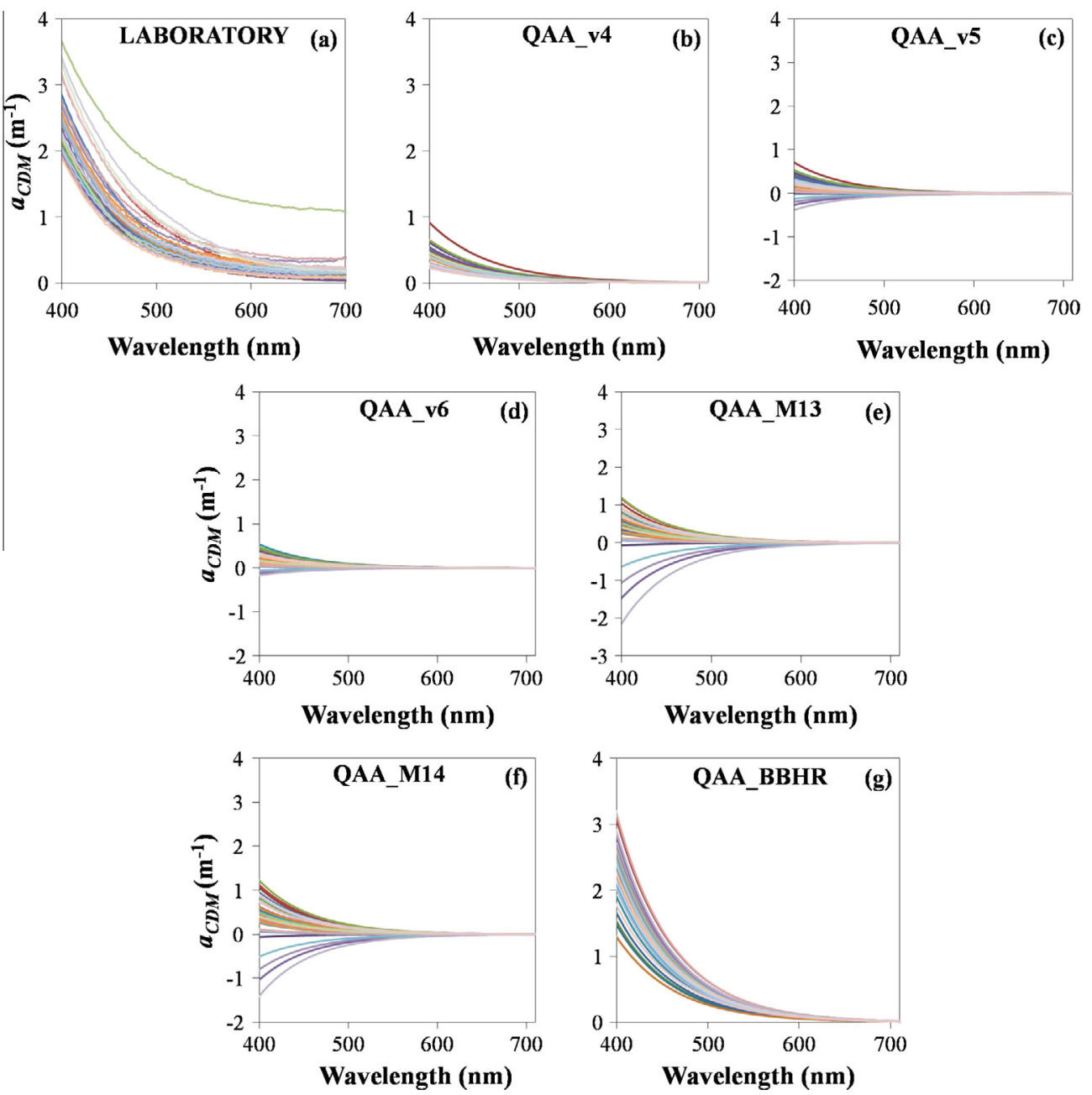

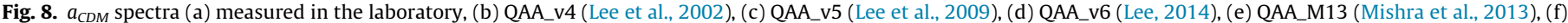
QAA_M14 (Mishra et al., 2014) and (g) QAA_BBHR.

QAA_M14 do not use fixed $S$ values, they use models that consider an intercept of the function that equate to a value of $S$ around 0.015 . This value is adopted because studies have shown that $S$ varies between 0.01 and $0.02 \mathrm{~nm}^{-1}$ (Hoogenboom et al., 1998); hence, the average value of $0.015 \mathrm{~nm}^{-1}$ is used (Lee et al., 2002; Lee and Carder, 2004). However, this value is considerably high when compared to minimum values of $S$ obtained from laboratory measurements of $a_{C D M}$ in BBHR, yielding a NRMSE of $15.5 \%$. A magnitude difference of 0.1 was observed between the averaged measured and estimated $S$, with a NRMSE of $15.5 \%$. In this study, the intercept value was replaced with 0.014 , which yielded better results, deriving an average $S$ of $0.016 \mathrm{~nm}^{-1}$, with a low NRMSE of $14.4 \%$.

QAA_BBHR produced negative values of $a_{C D M}(\lambda)$ even after the calibration of $\xi$. Hence, Eq. (15) was also tuned to improve the estimation of $\zeta$. QAA versions tested in this research yielded an average of 0.91 for $\zeta$ which is much closer to the $\zeta$ produced by $a_{\varphi}(411) /$ $a_{\varphi}(443)$ ratio using $a_{\varphi}$ obtained in laboratory. According to Mishra et al. (2014), underestimated values of $\zeta$ can lead to underestimation of $a_{C D M}(\lambda)$; however, the opposite was observed. $\zeta$ derived from $a_{\varphi}(411) / a_{\varphi}(443)$ ratio yielded negative values of $a_{C D M}(\lambda)$. Therefore, we concluded that the previously used ratio of $a_{\varphi}(411) / a_{\varphi}(443)$ may not be suitable to represent the pigments in inland waters. Combinations of wavelengths were tested and $a_{\varphi}(665) / a_{\varphi}(709)$ emerged as the ratio that generated lowest NRMSE and MAPE. The new ratio produced an average $\zeta$ of about
0.50 and adjustments were made in QAA_BBHR to estimate $\zeta$ accurately. These modifications to $\zeta$ estimation improved the prediction accuracy of $a_{C D M}(\lambda)$ and, consequently, $a_{\varphi}(\lambda)$.

Fig. 9 shows the plots of the measured versus estimated $a_{C D M}$ at $443 \mathrm{~nm}$, a wavelength commonly used in CDOM prediction. Although $a_{C D M}$ was retrieved for other MERIS/Sentinel-2A/3A bands, the result is not presented in the paper. All tested QAA versions underestimated $a_{C D M}(\lambda)$, with negative average bias varying from $-0.58 \mathrm{~m}^{-1}$ (QAA_v5 and QAA_v6) to $-0.53 \mathrm{~m}^{-1}$ (QAA_v4). $412 \mathrm{~nm}$ produced the highest bias, due to uncorrected fit of $\zeta$ and $\xi$, while $709 \mathrm{~nm}$ showed the lowest bias. Although QAA_v4 did not produce negative $a_{C D M}(\lambda)$, this version showed a considerably high average bias of $-0.53 \mathrm{~m}^{-1}$, with maximum of $-1.67 \mathrm{~m}^{-1}$ at $412 \mathrm{~nm}$, and minimum of $-0.14 \mathrm{~m}^{-1}$ at $709 \mathrm{~nm}$. QAA_M13 and QAA_M14 versions presented intermediate average bias of -0.55 and $-0.54 \mathrm{~m}^{-1}$, respectively.

The modification proposed in QAA_BBHR to estimate $\zeta$ and $\xi$ significantly improved the estimation of $a_{C D M}(\lambda)$. QAA_BBHR produced a considerably low average bias of $-0.03 \mathrm{~m}^{-1}$ and did not show underestimation at 443, 510 and $560 \mathrm{~nm}$ unlike other versions. Fig. 9f highlights a systematic error rated to sampling location P7, collected in October 2014, which exhibited higher underestimation at every wavelength and created a line almost parallel to 1:1 line. It was not possible to isolate bias observed for P7 in other QAA versions because of the overall severe underes- 

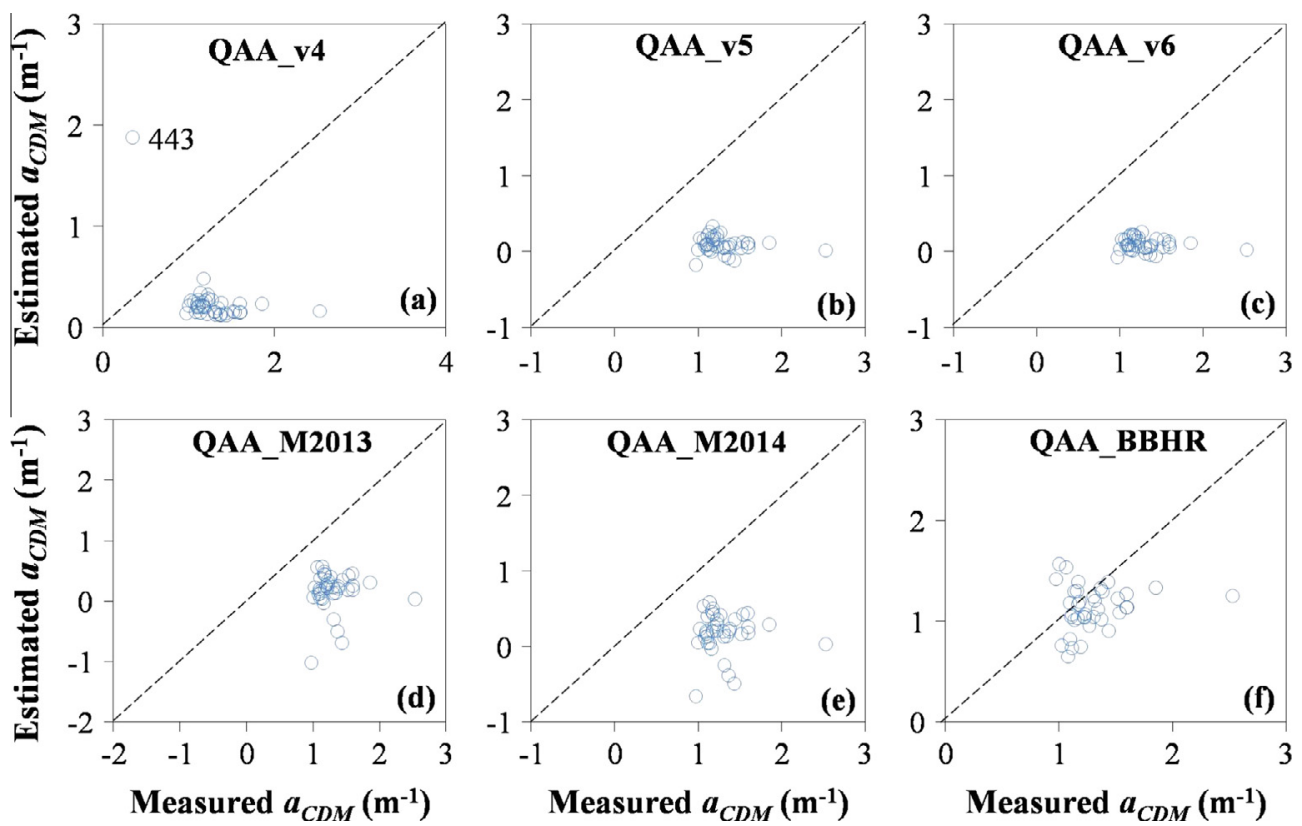

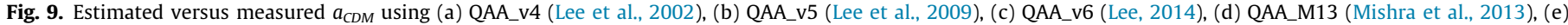
QAA_M14 (Mishra et al., 2014) and (f) QAA_BBHR.

timation. P7 measured in the laboratory presented a different behavior from other samples, with very high $a_{C D M}(\lambda)$ values and a low $S$ of $0.0124 \mathrm{~nm}^{-1}$, while the mean was of $0.0161 \mathrm{~nm}^{-1}$. Eliminating the sample P7 and recalculating the bias substantially improved the errors; however, the removal of P7 at the calibration worsened the QAA_BBHR fit.

Fig. 10 shows the NRMSE and MAPE obtained for $a_{C D M}$ for each QAA version. QAA_BBHR showed a consistently lower NRMSE and MAPE compared to other QAA versions, whereas, QAA_v4, QAA_v5, QAA_v6, QAA_M13 and QAA_M14 produced similar error pattern. Higher NRMSE was observed at shorter wavelengths (412 and $443 \mathrm{~nm}$ ) and higher MAPE at longer wavelengths $(709 \mathrm{~nm})$, due to severe underestimation of these spectral regions. The negative values produced at shorter wavelengths reflected considerably in errors obtained for other IOPs. Among the existing tested models, none yielded a MAPE lower than $88 \% . a_{C D M}(\lambda)$ estimation accuracy by QAA_v4 was comparatively higher than other models with a mean NRMSE of $42.39 \%$ and MAPE of $88.16 \%$ because it did not generate negative values. QAA_v5 was the least accurate in estimating $a_{C D M}(\lambda)$ among all algorithms, resulting an average NRMSE of $45.54 \%$ and MAPE of $95.46 \%$ across the visible spectrum; however, it produced good estimates at longer wavelengths, with NRMSE of $22.08 \%$ at $681 \mathrm{~nm}$ and $24.38 \%$ at $620 \mathrm{~nm}$.

The algorithms for inland waters, QAA_M13 and QAA_M14, also produced similar errors values, with an average NRMSE of up to $44.52 \%$ and MAPE up to $92.12 \%$. These versions also presented similar error patterns to QAA versions for marine waters, because they also yielded negative values of $a_{C D M}(\lambda)$. As expected, the highest NRMSE was observed at $412 \mathrm{~nm}(106.03 \%)$ and lowest at $665 \mathrm{~nm}$ (22.03\%).

On the contrary, MAPE presented higher values at longer wavelengths. Divergence between NRMSE and MAPE occurs when the difference of estimated and measured values is high and the variance of the observations also is high, i.e., when the measured value is close to minimum value. In this case, such behavior is associated mainly with variance observed in $a_{C D M}(\lambda)$ caused by high $a_{C D M}(\lambda)$ at P7. QAA_BBHR was successful in producing accurate estimation of $a_{C D M}(\lambda)$ with an average NRMSE of $21.71 \%$ and MAPE of $47.41 \%$. The best results were obtained in the red region, with a NRMSE of
$20.3 \%$ at $620 \mathrm{~nm}$ and $20.2 \%$ at $665 \mathrm{~nm}$. Shorter wavelengths produced higher errors, mainly at $412 \mathrm{~nm}$ where NRMSE was $27.5 \%$ and MAPE $18.23 \%$.

\section{4. $a_{\varphi}(\lambda)$ retrieval}

Fig. 11 shows the $a_{\varphi}$ spectra estimated by spectrophotometer (Fig. 11a), QAA_v4 (Fig. 11b), QAA_v5 (Fig. 11c), QAA_v6 (Fig. 11d), QAA_M13 (Fig. 11e), QAA_M14 (Fig. 11f) and QAA_BBHR (Fig. 11g). An accurate estimation of $a_{\varphi}(\lambda)$ is entirely dependent on estimation accuracies of $a_{t}(\lambda)$ and $a_{C D M}(\lambda)$. In addition, errors in estimating $\zeta$ and $\xi$ can result in large errors of estimation for $a_{C D M}(\lambda)$ and $a_{\varphi}(\lambda)$, generating negative or zero values (Lee et al., 2010). The underestimation of $a_{t}(\lambda)$ by QAA_v4, QAA_v5 and QAA_v6 consequently underestimated $a_{\varphi}(\lambda)$ producing negative values, while QAA_M13 and QAA_M14 overestimated $a_{t}(\lambda)$ and $a_{\varphi}(\lambda)$. The main problem of existing versions was the estimation of $\zeta$ and $\xi$. Therefore, such values ( $\zeta$ and $\xi$ ) were fitted considering bio-optical status of the BBHR, where the red spectral region exhibited higher $a_{\varphi}(\lambda)$ contribution to $a_{t}(\lambda)$ than the blue spectral region, due to high influence of CDOM at shorter wavelengths. $\xi$ was calibrated in relation to $S$ (spectral slope of CDOM) observed in BBHR, while $\zeta$ was parametrized in order to represent better the pigments variation; in other hands, $a_{\varphi}(411) / a_{\varphi}(443)$ ratio was replaced with $a_{\varphi}(665) /$ $a_{\varphi}(709)$ ratio to define $\zeta$. The revised fits of $\zeta$ and $\xi$ improved the estimation of $a_{C D M}(\lambda)$ and $a_{\varphi}(\lambda)$ significantly. Even then, QAA_BBHR still produced an underestimation of $a_{\varphi}(\lambda)$ at shorter wavelengths such as 412 and $443 \mathrm{~nm}$, and at $709 \mathrm{~nm}$ (Fig. 11g).

Measured versus estimated $a_{\varphi}(\lambda)$ plots for wavelengths at 412 , 443, 620 and $665 \mathrm{~nm}$, the wavelengths associated with phytoplankton pigment absorption and, commonly used in pigment prediction, are shown in Fig. 12. As expected, all QAA versions developed for open ocean waters (QAA_v4, QAA_v5, and QAA_v6) underestimated $a_{\varphi}(\lambda)$, since $a(\lambda)$ already was lower than in situ $a_{\varphi}(\lambda)$.

QAA_v4 and QAA_v5 retrieved negative $a_{\varphi}(\lambda)$ values at longer wavelengths. However, the negative predictions were not restricted to algorithms developed for coastal and ocean waters only. QAA_turbid proposed by Yang et al. (2013) for turbid lakes 

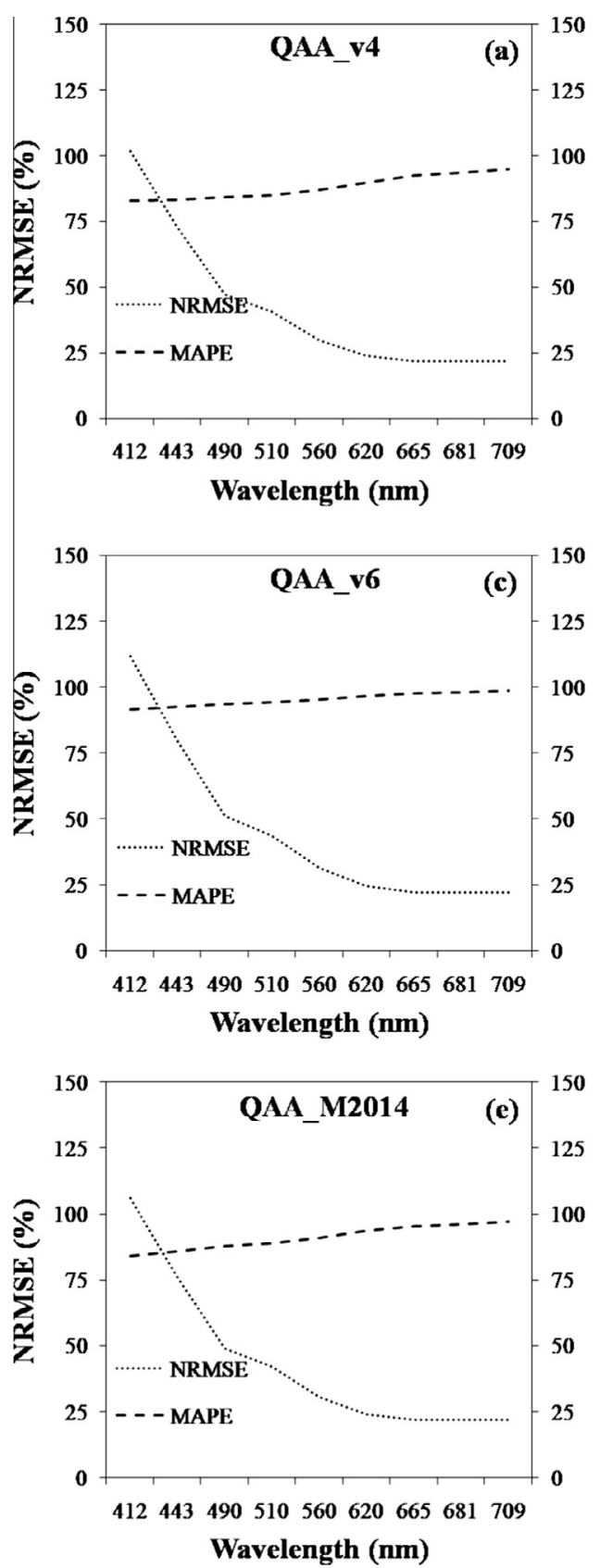
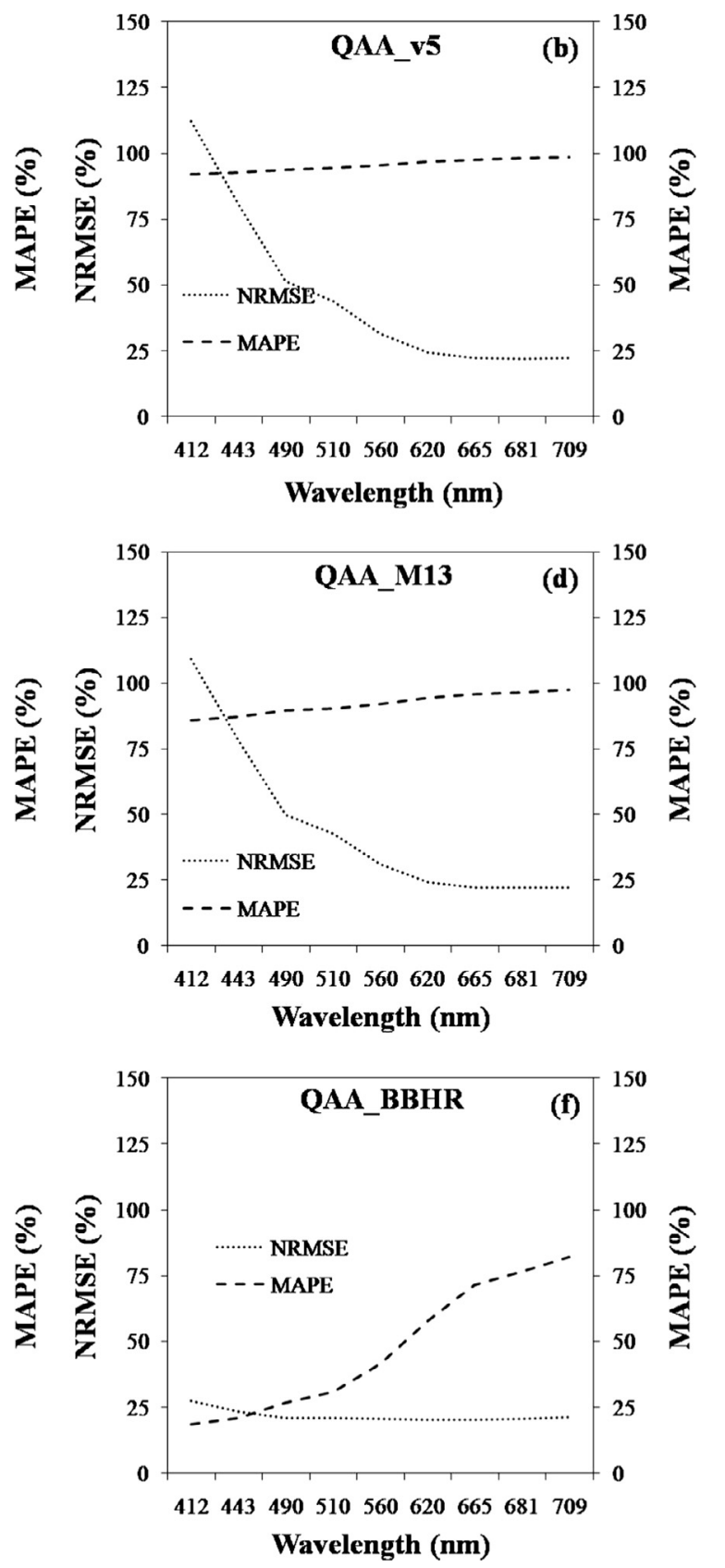

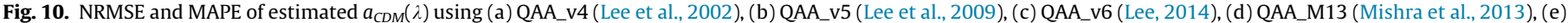
QAA_M14 (Mishra et al., 2014) and (f) QAA_BBHR.

also produced negative estimations at $443 \mathrm{~nm}$. These results demonstrate that $a_{\varphi}$ retrieval is affected by the bio-optical state of the water body and reinforce the importance of calibration of the empirical steps in a QAA. The empirical steps in these algorithms were calibrated for sites with Chl-a concentration much lower than BBHR and, therefore, did not produce accurate estimations in BBHR. Among the three versions for ocean waters, QAA_v5 showed the least underestimation, with an average bias of $-0.46 \mathrm{~m}^{-1}$ and a bias range of $-0.94 \mathrm{~m}^{-1}(412 \mathrm{~nm})$ to $-0.05 \mathrm{~m}^{-1}$ $(560 \mathrm{~nm})$. On the other hand, among the versions parametrized for inland waters, QAA_M13 showed the highest overestimation of $a_{\varphi}(\lambda)$ at every wavelength with a large average bias of $1.2 \mathrm{~m}^{-1}$, maximum of $1.69 \mathrm{~m}^{-1}$ at $443 \mathrm{~nm}$ and minimum of $0.36 \mathrm{~m}^{-1}$ at $709 \mathrm{~nm}$. QAA_M14 also overestimated $a_{\varphi}$ at every wavelength with a high average bias of $0.95 \mathrm{~m}^{-1}$.
Overall, QAA_BBHR showed a slight underestimation with an average bias of $-0.08 \mathrm{~m}^{-1}$ mainly due to the low bias of $-1.13 \mathrm{~m}^{-1}$ observed at $412 \mathrm{~nm}$. Sample P3 collected in October 2014 produced the highest underestimation at 412 and $443 \mathrm{~nm}$ as observed in Fig. 12f. P3 exhibited the highest Chl- $a$ concentration of $797.8 \mathrm{mg} \mathrm{m}^{-3}$ and, consequently, the highest in situ $a_{\varphi}(\lambda)$. Likely, the calibration of QAA_BBHR using the sample mean was not capable of estimating accurately the extreme values of $a_{\varphi}(\lambda)$. Other sample significantly underestimated was P14 collected also in October, with the second highest Chl- $a$ concentration of $723.5 \mathrm{mg} \mathrm{m}^{-3}$ and high $a_{\varphi}(\lambda)$. The main peculiarities of $a_{\varphi}(\lambda)$ for this sample is the greatest gradient between 412 and $443 \mathrm{~nm}$, and the median absorption at $665 \mathrm{~nm}$ compared to samples with lower concentrations. 

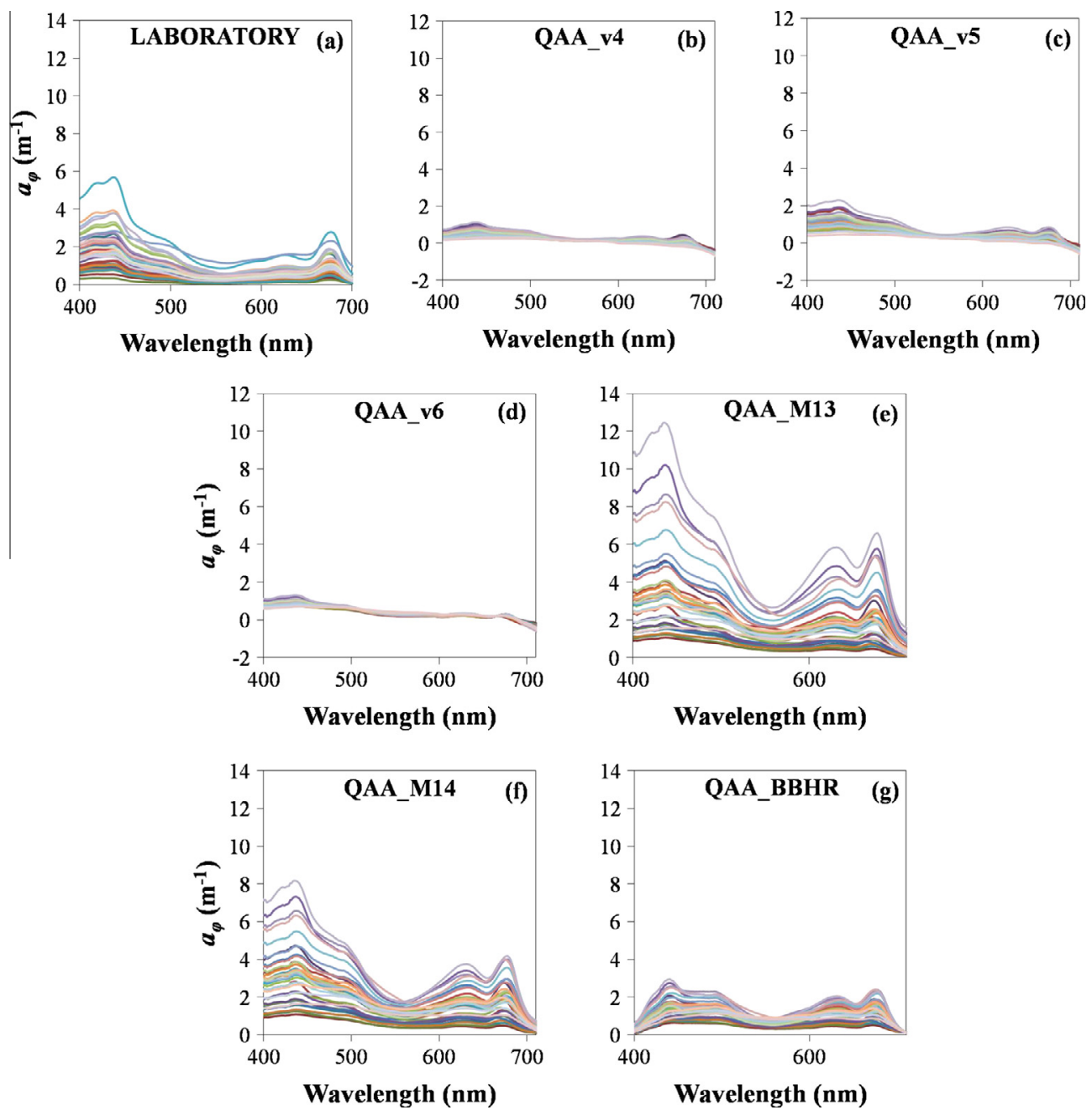

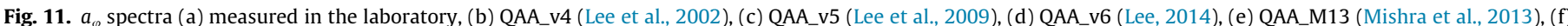
QAA_M14 (Mishra et al., 2014) and (g) QAA_BBHR.
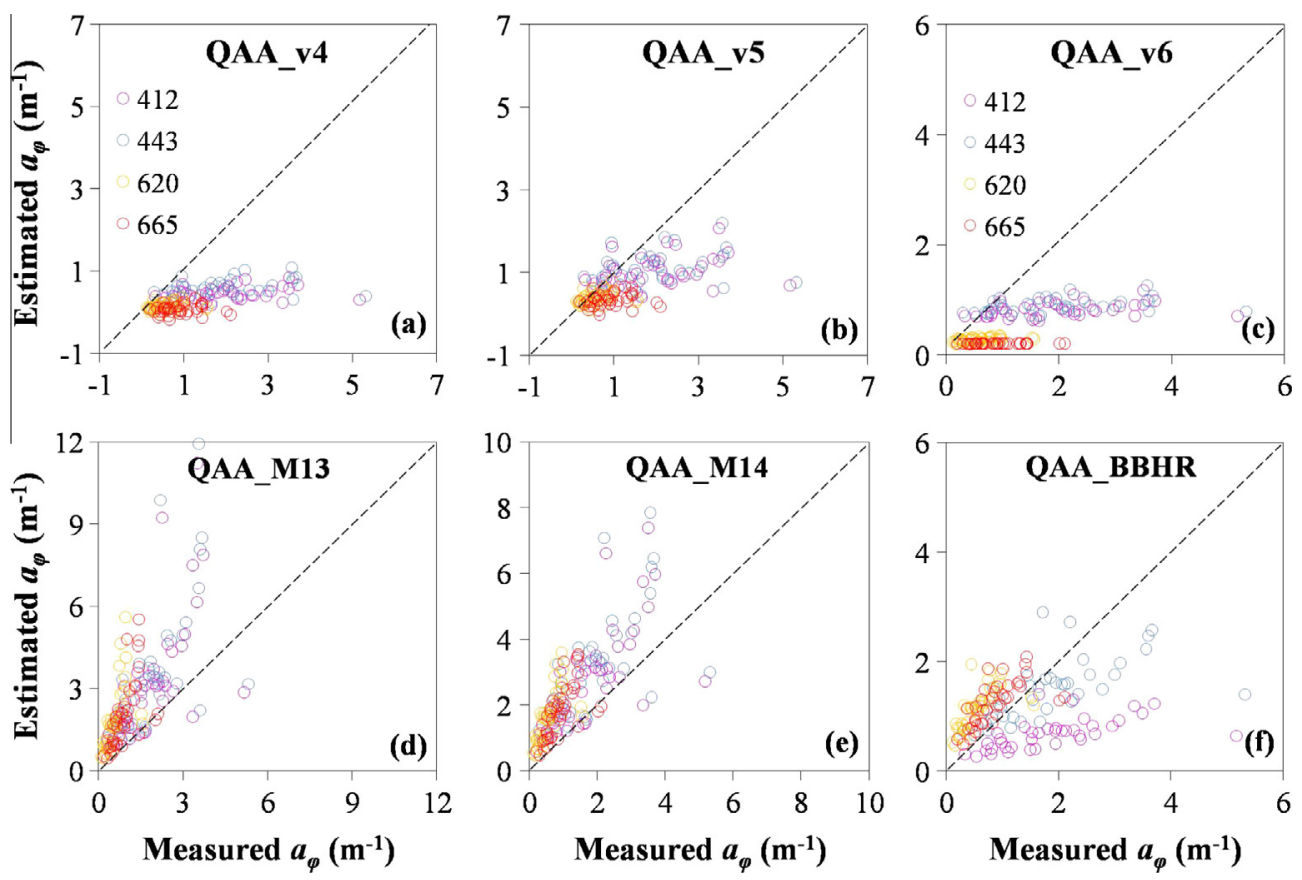

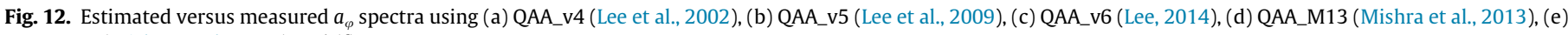
QAA_M14 (Mishra et al., 2014) and (f) QAA_BBHR. 

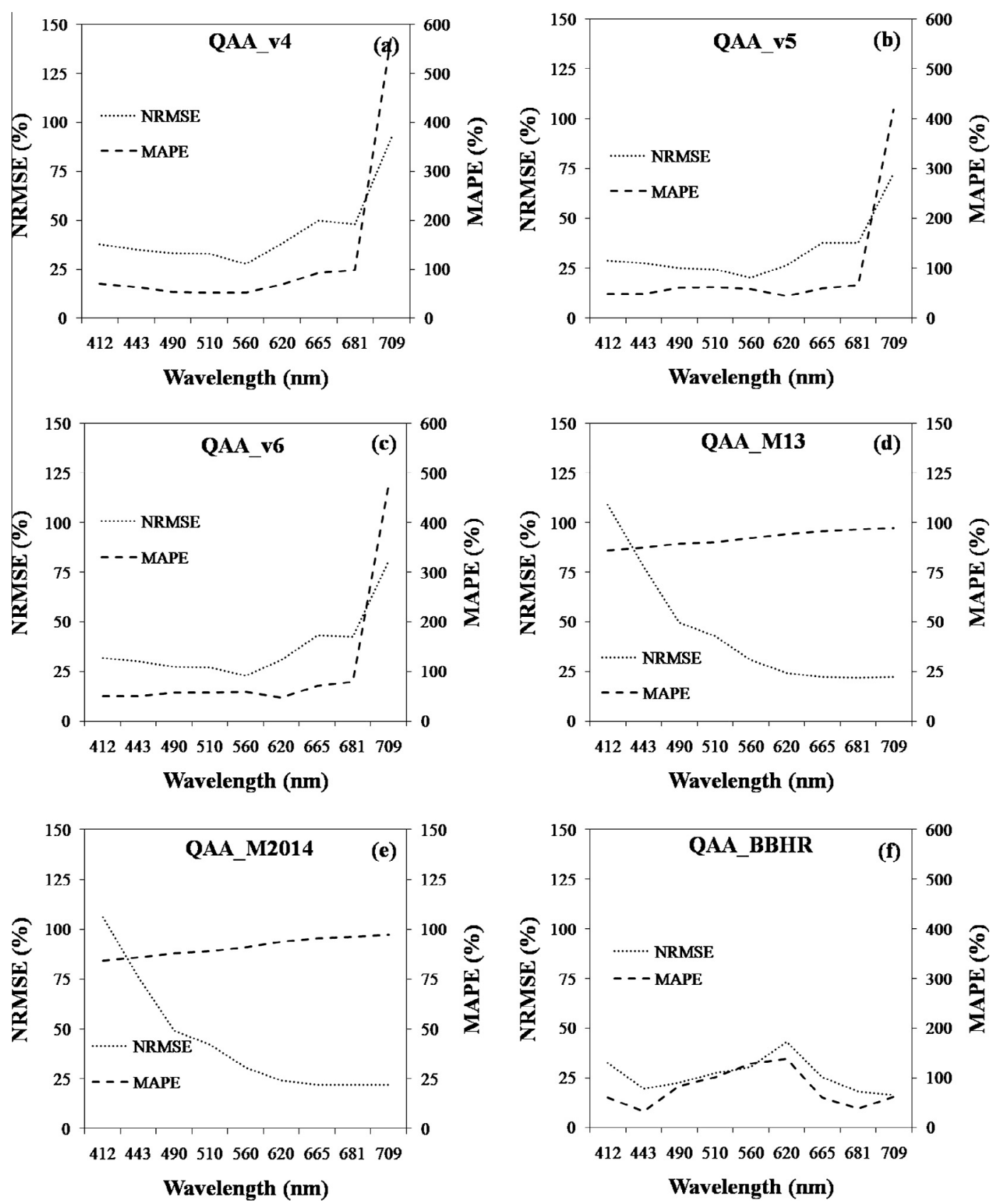

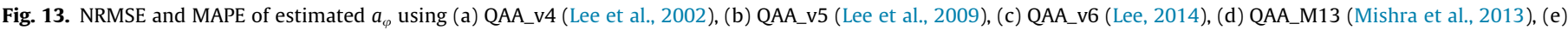
QAA_M14 (Mishra et al., 2014) and (f) QAA_BBHR.

Fig. 13 shows the NRMSE and MAPE obtained for $a_{\varphi}(\lambda)$ using QAA_v4 (Fig. 13a), QAA_v5 (Fig. 13b), QAA_v6 (Fig. 13c), QAA_M13 (Fig. 13d), QAA_M14 (Fig. 13e) and QAA_BBHR (Fig. 13f). QAA_v4, QAA_v5 and QAA_v6 showed considerably low NRMSE and MAPE, except at $709 \mathrm{~nm}$. Among the three versions for ocean waters, QAA_v5 showed the lowest errors and QAA_v6 yielded the highest average errors. Surprisingly, QAA_M13 and QAA_M14 presented higher NRMSE and MAPE compared to open ocean versions, due to overestimation, mainly at intermediate wavelengths. Among all five previous QAA versions, QAA_M13 presented the highest average NRMSE (77.63\%) and MAPE (182.91\%). With the exception of 412 and $443 \mathrm{~nm}$, every wavelength presented NRMSE higher than $65 \%$ with maximum value of $113 \%$ at $620 \mathrm{~nm}$ as well as MAPE range of $88.83 \%(412 \mathrm{~nm})$ to $278.47 \%(709 \mathrm{~nm})$.

Similarly, QAA_M14 yielded a high average NRMSE of $56.25 \%$ and MAPE of $163.32 \%$. Overall, QAA_BBHR considerably improved the accuracy estimation of $a_{\varphi}(\lambda)$ with an average NRMSE of $26.08 \%$ and MAPE of $78.08 \%$. The most accurate results were obtained at longer wavelengths such as $681 \mathrm{~nm}$ (NRMSE: $18.02 \%$ and MAPE: $37.5 \%$ ), with a slight improvement at shorter wavelengths (NRMSE: 19.6\% and MAPE: $32.8 \%$ for $443 \mathrm{~nm}$ ). High errors were observed at intermediate wavelengths, with maximum at $620 \mathrm{~nm}$ (NRMSE: 43.1\% and MAPE: 138.1\%).

\subsection{Chl-a retrieval from $a_{\varphi}(\lambda)$}

To further assess the $a_{\varphi}(\lambda)$, some Chl- $a$ models were reparametrized using $a_{\varphi}(\lambda)$ and $a_{w}(\lambda)$, and validated and compared with their $R_{r s}$ counterparts. The 2B (Gitelson et al., 2003), 3B (Gitelson et al., 2008), and NDCI (Mishra and Mishra, 2012) Chl-a models were tested. The $a_{\varphi}(\lambda)$ and $a_{w}(\lambda)$ based models representing the $2 \mathrm{~B}, 3 \mathrm{~B}$, and NDCI were labeled as $\Psi_{1}, \Psi_{2}$ and $\Psi_{3}$ as shown in 

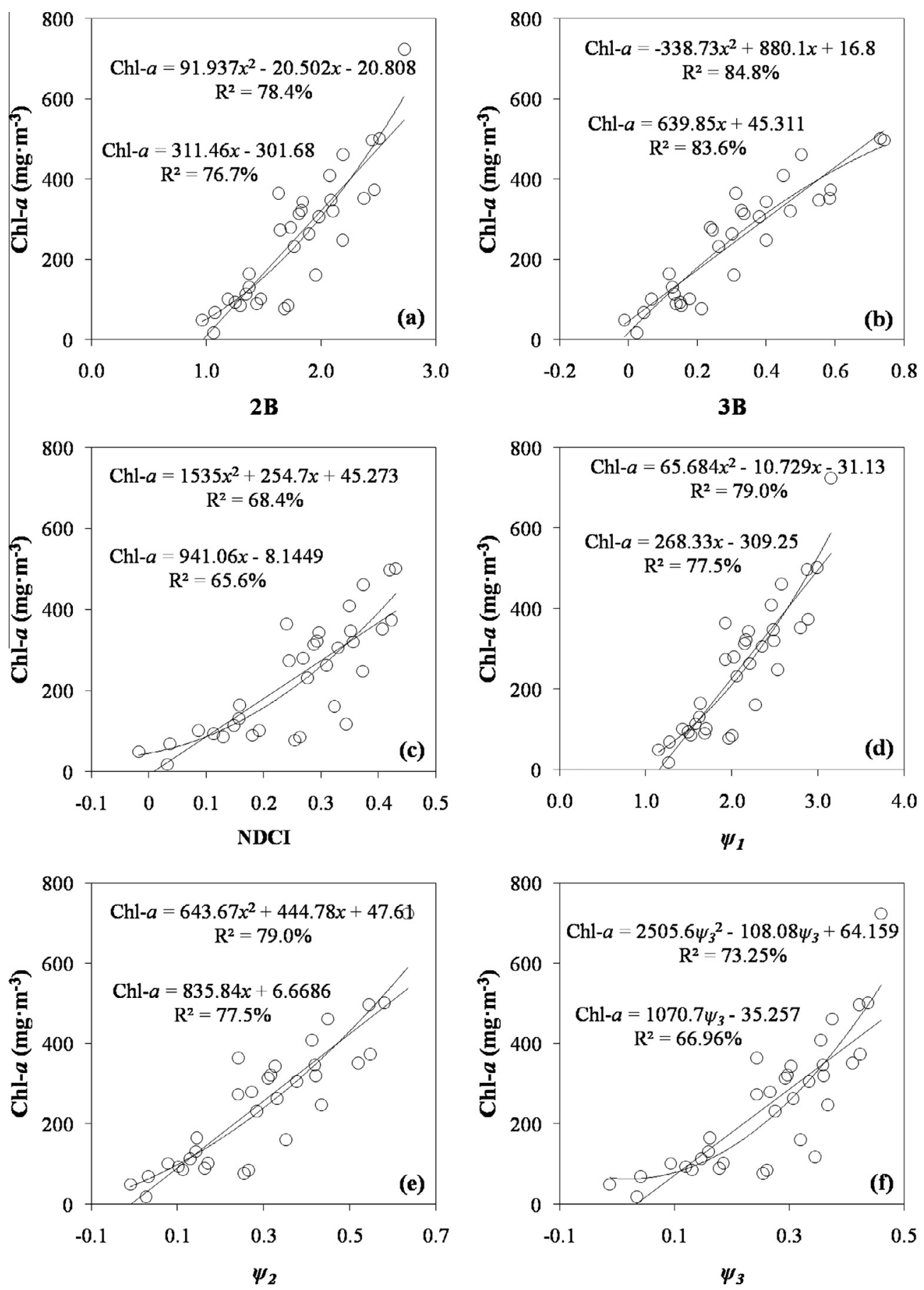

Fig. 14. Scatter plot showing empirical fit between Chl- $a$ and (a) $2 \mathrm{~B}$; (b) 3B; (c) NDCl; (d) $\Psi_{1}$; (e) $\Psi_{2}$; and (f) $\Psi_{3}$.

Eqs. (22), (24), and (26). Fig. 14 shows the fit of the models for 2B (Fig. 14a), 3B (Fig. 14b), NDCI (Fig. 14c), $\Psi_{1}$ (Fig. 14d), $\Psi_{2}$ (Fig. 14e), and $\Psi_{3}$ (Fig. 14f). The indices using $R_{r s}$ exhibited high correlation with the Chl-a, e.g., 0.88 for $2 \mathrm{~B}, 0.91$ for $3 \mathrm{~B}$, and 0.81 for NDCI, while the indices based on $a_{\varphi}(\lambda)$ and $a_{w}(\lambda)$ presented correlation of 0.88 for $\Psi_{1}, 0.88$ for $\Psi_{2}$, and 0.81 for $\Psi_{3}$. With exception of $\Psi_{2}$, models based on QAA $a_{\varphi}(\lambda)$ and $a_{w}(\lambda)$ provided equal correlation with Chl- $a$ compared to their $R_{r s}$ counterparts. This serves as an indirect validation of the QAA_BBHR and the derived IOPs.

Table 3 shows the calibration results in terms of standard error of estimation (S), determination coefficient $\left(R^{2}\right.$, in \%), adjusted $R^{2}$
$\left(\operatorname{Adj}-R^{2}\right.$, in \%), $F$ statistic $(F)$, and $p$-value. Most of the calibration variants produced significant results with $R^{2}$ of approximately 0.7 and $p$-value equal or close to zero. The linear fits were statistically more significant than quadratic fits, with the highest $F$ values.

To assess the performance of the fitted models validation was carried out using a dataset collected on September, 2015. Table 4 shows the validation results of the Chl- $a$ estimation models in terms of RMSE, NRMSE, MAPE, bias and $R^{2}$ between measured and estimated Chl- $a$. The quadratic adjustment was more suitable for $2 \mathrm{~B}$ and $\mathrm{NDCI}$, producing least errors, whereas, the linear fit was better for 3B index. Among all the models, $\Psi_{1}$ exhibited the best performance, with $\mathrm{NRMSE}=13.84 \%$ and $\mathrm{MAPE}=16.22 \%$, while 
Table 3

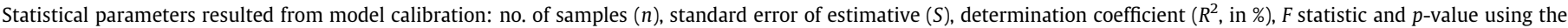
indices $2 \mathrm{~B}, 3 \mathrm{~B}, \mathrm{NDCI}, \Psi_{1} \Psi_{2}$ and $\Psi_{3}$ for retrieving the Chl- $a$ concentration.

\begin{tabular}{|c|c|c|c|c|c|c|c|}
\hline Index & $n$ & Fit & $S$ & $R^{2}$ & $\operatorname{Adj}-R^{2}$ & $\mathrm{~F}$ & $p$-value \\
\hline \multicolumn{8}{|c|}{ Models calibrated using $R_{r s}$} \\
\hline $2 \mathrm{~B}$ & 33 & Linear & 79.82 & 76.7 & 76.0 & 102.15 & 0.000 \\
\hline $2 B$ & 33 & Quadratic & 78.24 & 78.3 & 76.9 & 54.28 & 0.000 \\
\hline 3B & 31 & Linear & 57.94 & 83.6 & 83.0 & 147.96 & 0.000 \\
\hline 3B & 31 & Quadratic & 56.89 & 84.7 & 83.7 & 77.78 & 0.000 \\
\hline $\mathrm{NDCI}$ & 33 & Linear & 83.68 & 65.6 & 64.5 & 59.13 & 0.000 \\
\hline $\mathrm{NDCI}$ & 33 & Quadratic & 81.58 & 68.4 & 66.3 & 32.42 & 0.000 \\
\hline \multicolumn{8}{|c|}{ Models using $a_{\varphi}$ and $a_{w}$ derived from $Q A A \_B B H R$} \\
\hline$\Psi_{1}$ & 33 & Linear & 78.40 & 77.5 & 76.8 & 107.00 & 0.000 \\
\hline$\Psi_{1}$ & 33 & Quadratic & 77.06 & 79.0 & 77.6 & 56.43 & 0.000 \\
\hline$\Psi_{2}$ & 33 & Linear & 78.42 & 77.5 & 76.8 & 106.9 & 0.000 \\
\hline$\Psi_{2}$ & 33 & Quadratic & 77.02 & 79.0 & 77.6 & 56.51 & 0.000 \\
\hline$\Psi_{3}$ & 34 & Linear & 87.99 & 71.7 & 70.8 & 78.56 & 0.000 \\
\hline$\Psi_{3}$ & 34 & Quadratic & 79.32 & 77.7 & 76.3 & 52.40 & 0.000 \\
\hline
\end{tabular}

Table 4

Validation of the Chl- $a$ estimation models using RMSE, NRMSE, MAPE, bias and $R^{2}$. The best results were highlighted in bold.

\begin{tabular}{|c|c|c|c|c|c|c|}
\hline Index & Fit & $\operatorname{RMSE}\left(\mathrm{mg} \mathrm{m}^{-3}\right)$ & NRMSE (\%) & MAPE (\%) & $\operatorname{Bias}\left(\mathrm{mg} \mathrm{m}^{-3}\right)$ & $R^{2}(\%)$ \\
\hline \multicolumn{7}{|c|}{ Models calibrated using $R_{r s}$} \\
\hline $2 \mathrm{~B}$ & Linear & 101.46 & 55.46 & 88.58 & -99.27 & 83.67 \\
\hline $2 B$ & Quadratic & 69.06 & 37.75 & 51.48 & -64.21 & 82.93 \\
\hline $3 B$ & Linear & 69.35 & 37.91 & 57.74 & -66.41 & 84.14 \\
\hline $3 \mathrm{~B}$ & Quadratic & 94.53 & 51.68 & 83.94 & -91.09 & 83.57 \\
\hline $\mathrm{NDCI}$ & Linear & 118.60 & 64.83 & 107.32 & -114.28 & 82.65 \\
\hline $\mathrm{NDCI}$ & Quadratic & 72.15 & 39.44 & 53.27 & -67.01 & 80.41 \\
\hline \multicolumn{7}{|c|}{ Models using $a_{\varphi}$ and $a_{w}$ derived from QAA_BBHR } \\
\hline$\Psi_{1}$ & Linear & 94.59 & 51.71 & 81.91 & -91.79 & 81.03 \\
\hline$\Psi_{1}$ & Quadratic & 25.31 & 13.84 & 16.22 & 1.01 & 80.91 \\
\hline$\Psi_{2}$ & Linear & 95.01 & 51.94 & 82.47 & -92.30 & 81.67 \\
\hline$\Psi_{2}$ & Quadratic & 66.24 & 36.21 & 49.25 & -61.3 & 81.47 \\
\hline$\Psi 3$ & Linear & 141.91 & 77.58 & 129.11 & -134.44 & 79.0 \\
\hline$\Psi_{3}$ & Quadratic & 71.37 & 39.15 & 41.95 & -47.50 & 0.59 \\
\hline
\end{tabular}

Table 5

Comparison between empirical steps of the QAA_BBHR and QAA_v5 to derive IOPs from $R_{r s}$.

\begin{tabular}{|c|c|c|}
\hline$u=b_{b} /\left(a+b_{b}\right)$ & $\begin{array}{l}r_{r s}(\lambda)=R_{r s}(\lambda) /\left(0.52+1.7 R_{r s}(\lambda)\right) \\
r_{r s}(\lambda)=\left(g_{0}+g_{1} u(\lambda)\right) u(\lambda) \\
u(\lambda)=\frac{-g_{0}+\sqrt{\left(g_{0}\right)^{2}+4 g_{1} r_{r s}(\lambda)}}{2 g_{1}} \\
g_{0}=0.089, g_{1}=0.125 \\
\text { QAA_BBHR }\end{array}$ & QAA_v5 \\
\hline \multirow[t]{3}{*}{$a\left(\lambda_{0}\right)$} & $\lambda_{0}=709 \mathrm{~nm}$ & $\lambda_{0}=555 \mathrm{~nm}$ \\
\hline & 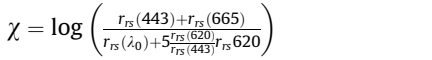 & $\chi=\log \left(\frac{r_{r s}(443)+r_{r s}(490)}{r_{r s}\left(\lambda_{0}\right)+5_{\frac{r_{r s}(6667}{r_{5}(490)} r_{r_{s}}(667)}}\right)$ \\
\hline & $a\left(\lambda_{0}\right)=a_{w}\left(\lambda_{0}\right)+10^{-0.7702+0.0999 \chi+0.0566 \chi^{2}}$ & $a\left(\lambda_{0}\right)=a_{w}\left(\lambda_{0}\right)+10^{-1.146-1.366 \gamma-0.469 \chi^{2}}$ \\
\hline$\eta$ & $\eta=2.0\left(1-1.2 \exp \left(-0.9 \frac{r_{r s}(443)}{r_{r s}(555)}\right)\right)$ & $\eta=2.0\left(1-1.2 \exp \left(-0.9 \frac{r_{r s}(443)}{r_{r s}(555)}\right)\right)$ \\
\hline \multirow[t]{2}{*}{$\zeta$} & $\zeta=a_{\varphi}(665) / a_{\varphi}(709)$ & $\zeta=a_{\varphi}(411) / a_{\varphi}(443)$ \\
\hline & $\zeta=0.3+\frac{0.2}{0.8+r_{r s}(665) / r_{r s}(709)}$ & $\zeta=0.74+\frac{0.2}{0.8+r_{r 5}(443) / r_{r s}\left(\lambda_{0}\right)}$ \\
\hline \multirow[t]{2}{*}{$\xi$} & $\begin{array}{l}\xi=a_{C D M}(411) / a_{C D M}(443) \\
\xi=e^{S(443-411)}\end{array}$ & $\begin{array}{l}\xi=a_{C D M}(411) / a_{C D M}(443) \\
\xi=e^{S(443-411)}\end{array}$ \\
\hline & $S=0.014+\frac{0.002}{0.6+r_{r s}(443) / r_{r s}\left(\lambda_{0}\right)}$ & $S=0.015+\frac{0.002}{0.6+r_{r s}(443) / r_{r s}\left(\lambda_{0}\right)}$ \\
\hline
\end{tabular}

the second best performance (2B) presented NRMSE $=35.32 \%$ and MAPE $=44.33 \%$. The improvement shown by $\Psi_{1}$ compared to $2 \mathrm{~B}$ is likely associated with the efficiency of the QAA_BBHR in minimizing the influence of absorption by other ORCs, not totally removed by $R_{r s}(709) / R_{r s}(665)$ ratio. The incorporation of a third wavelength $(754 \mathrm{~nm})$ did not improve the $3 \mathrm{~B}$ or $\Psi_{2}$ performance in relation to $2 \mathrm{~B}$ and $\Psi_{1}$. Overall, $754 \mathrm{~nm}$ is inserted to minimize backscattering influence in Chl- $a$ estimating (Gitelson et al., 2008). Overall, quadratic NDCI, quadratic 2B and linear 3B exhibited similar errors; despite this, a $t$-student test for paired samples showed that models are not statistically equal.

\section{Conclusion}

Different QAA versions have been developed for different environments (ocean, coastal waters and highly productive ponds) to exploit the range the greater variability of ORCs concentrations; however, these QAA versions were not able to estimate IOPs accurately in a tropical eutrophic reservoir. The results derived from testing the aforementioned models clearly indicated the need for re-parametrization. Thereby, QAA_BBHR was developed in this study to address the gap in performance of QAAs in eutrophic reservoirs. This study shows a critical breakdown and comprehen- 
sive comparisons of all existing variants of QAAs. The new parameterization and calibration developed in QAA_BBHR exhibited satisfactory results in estimating $a(\lambda), a_{C D M}(\lambda)$ and $a_{\varphi}(\lambda)$ in a tropical eutrophic reservoir.

The fine tuning and recalibration of the empirical steps of the existing QAAs considerably improved the prediction of IOPs. The use of $709 \mathrm{~nm}$ and the calibration of $\chi$ increased the estimation accuracy of $a\left(\lambda_{0}\right)$ and, consequently, accuracies of $a_{C D M}(\lambda)$ and $a_{\varphi}(\lambda)$ prediction. The novel parameterization of $\chi$ using the combination of $443,665,709,620$ and $443 \mathrm{~nm}$ and the adjustment of the calibration coefficients $\left(h_{0}, h_{1}\right.$ and $\left.h_{2}\right)$ were crucial changes in QAA_BBHR to estimate $a(\lambda)$ (Table 5). The use of $r_{r s}$ at 620 and $665 \mathrm{~nm}$ exhibited a better performance than $r_{r s}(665) \mathrm{nm}$ alone. Other important modifications were the calibration and parameterization of $\zeta$ and $\xi$. The changes carried out in these two steps were essential to reduce the severe underestimation (negative values) in $a_{C D M}(\lambda)$ and $a_{\varphi}(\lambda)$ observed before calibration. In addition, tests showed that $a_{\varphi}(\lambda)$ estimated by QAA_BBHR can be used successfully to retrieve Chl- $a$ concentration. The performance of models based on $a_{\varphi}(\lambda)$ and $a_{w}(\lambda)$ were better compared to their $R_{r s}$ counterparts. It is due to the fact that QAA_BBHR was successful in isolating the interference of other optically active components at phytoplankton absorption bands.

QAA_BBHR could be adopted as an option for frequent mapping the ORCs in Brazilian reservoirs and other similar environments around the world. The results obtained by QAA_BBHR showed that once calibrated the algorithm can be applied for monitoring purposes. QAA_BBHR can be scaled-up using bands of ESA's Sentinel$2 \mathrm{~A}$ and $3 \mathrm{~A}$ since the re-parametrization was done at MERIS band centers. Brazil and Sao Paulo State in particular has a tremendous need for a fast and inexpensive monitoring system for its cascade reservoirs which have been severely plagued by agriculture and urban runoff based nutrient pollution and persistent drought. QAA_BBHR provides a way forward to simultaneously resolve all ORCs from remotely sensed data.

\section{Acknowledgments}

The authors thank to São Paulo Research Foundation (FAPESP Process No. 2012/19821-1, 2013/09045-7, 2015/21586-9 and 2015/18525-8), National Council Scientific and Technological Development (CNPq Process No. 472131/2012-5 and 482605/2013-8), Science without Borders/CNPq (Process No. 400881/2013-6 and 200157/2015-9), and PPGCC/UNESP for financial support, Coordination for the Improvement of Higher Education Personnel (CAPES - Brazil) for scholarship. Thanks to the Office of International Education (OIE) and the Department of Geography at the University of Georgia (UGA) for facilitating the collaboration between the UNESP and UGA through the international student exchange program. The authors also thank Professor Edivaldo D. Velini and staffs from FCA/UNESP for allowing the use of their laboratory facilities.

\section{References}

AES Tietê, 2013. Barra Bonita <http://www.aestiete.com.br/usinas/Paginas/ BarraBonita.aspx>.

APHA, 1998. Standard Methods for the Examination of Water and Wastewater. American Public Health Association (APHA), American Water Works Association (AWWA), Water Environmental Federation (WEF), Washington, USA.

Brando, V.E., Dekker, A.G., 2003. Satellite hyperspectral remote sensing for estimating estuarine and coastal water quality. IEEE Trans. Geosci. Remote Sens. 41 (6), 1378-1387.

Bricaud, A., Morel, A., Prieur, L., 1981. Absorption by dissolved organic matter of the sea (yellow substance) in the UV and visible domains. Limnol. Oceanogr. 26 (1), 43-53.
Bricaud, A., Babin, M., Morel, A., Claustre, H., 1995. Variability in the chlorophyllspecific absorptions coefficients of natural phytoplankton: analysis and parameterization. J. Geophys. Res. 100 (C7), 13321-13332.

Calijuri, M.C., Santos, A.C.A., 1996. Short-term changes in the Barra Bonita reservoir (São Paulo, Brazil): emphasis on the phytoplankton communities. Hydrobiologia 330, 163-175.

Carder, K.L., Chen, F.R., Lee, Z.P., Hawes, S.K., Kamykowski, D., 1999. Semianalytical Moderate-Resolution Imaging Spectrometer algorithms for chlorophyll a and absorption with bio-optical domains based on nitrate-depletion temperatures. J. Geophys. Res. 104 (C3), 5403-5421.

Chen, J., Cui, T., Qiu, Z., Lin, C., 2014. A simple two-band semi-analytical model for retrieval of specific absorption coefficients in coastal waters. ISPRS J. Photogramm. Remote Sens. 91, 85-97.

Chen, S., Zhang, T., 2015. Evaluation of a QAA-based algorithm using MODIS land bands data for retrieval of IOPs in the Eastern China Seas. Opt. Express 23 (11), 13953-13971.

Ciotti, A.M., Lewis, M.R., Cullen, J.J., 2002. Assessment of the relationships between dominant cell size in natural phytoplankton communities and the spectral of the absorption coefficient. Limnol. Oceanogr. 47 (2), 404-417.

Cleveland, J.S., Weidemann, A.D., 1993. Quantifying absorption by aquatic particles: a multiple scattering correction for glass-fiber filters. Limnol. Oceanogr. 38 (6) 1321-1327.

Dellamano-Oliveira, M.J., Vieira, A.A.H., Rocha, O., Colombo, V., 2008. Phytoplankton taxonomic composition and temporal changes in a tropical reservoir. Fundam. Appl. Limnol. 171 (1), 27-38.

Doerffer, R., Schiller, H., 2007. The MERIS Case 2 water algorithm. Int. J. Remote Sens. 28 (3-4), 517-535.

ESA. European Space Agency, 2015. Missions <https://earth.esa.int/web/guest/ missions>.

Gitelson, A.A., Gritz, Y., Merzlyak, M.N., 2003. Relationships between leaf chlorophyll content and spectral reflectance and algorithms for nondestructive chlorophyll assessment in higher plant leaves. J. Plant Physiol. 160, 271-282.

Gitelson, A.A., Dall'Olmo, G., Moses, W., Rundquist, D.C., Barrow, T., Fisher, T.R., Gurlin, D., Holz, J., 2008. A simple semi-analytical model for remotes estimation of chlorophyll-a in turbid waters: validation. Remote Sens. Environ. 112, 3582 3593.

Golterman, H.L., 1975. Developments in Water Science 2. Physiological Limnology: An Approach to the Physiology of Lake Ecosystems. Elsevier, Amsterdam, Netherlands.

Goodin, D.G., Han, L., Fraser, R.N., Rundquist, D.C., Stebbins, W.A., Schalles, J.F., 1993. Analysis of suspended solids in water using remotely sensed high resolution derivative spectra. Photogramm. Eng. Remote Sens. 59 (4), 505-510.

Gordon, H.R., 1979. Diffuse reflectance of the ocean: the theory of its augmentation by chlorophyll a fluorescence at $685 \mathrm{~nm}$. Appl. Opt. 18 (8).

Gordon, H.R., Brown, O.B., Evans, R.H., Brown, J.W., Smith, R.C., Baker, K.S., Clark, D. K., 1988. A semianalytical radiance model of ocean color. J. Geophys. Res. 93, 10909-10924.

Gurlin, D., Gitelson, A.A., Moses, W.J., 2011. Remote estimation of chl-a concentration in turbid productive waters - return to a simple two-band NIRred model? Remote Sens. Environ. 115, 3479-3490.

Hoge, F.E., Lyons, P.E., 1996. Satellite retrieval of inherent optical properties by linear matrix inversion of oceanic radiance models: an analysis of model and radiance measurement errors. J. Geophys. Res. 101 (C7), 16631-16648.

Hoogenboom, H.J., Dekker, A.G., Althuis, I.A., 1998. Simulation of AVIRIS sensitivity for detecting chlorophyll over coastal and inland waters. Remote Sens. Environ. $65,333-340$.

IOCCG, 2006. Remote sensing of inherent optical properties: fundamentals, tests of algorithms, and applications. In: Lee, Z.P. (Ed.), Reports of the International Ocean-Colour Coordinating Group, 5. Dartmouth, Nova Scotia, Canada.

Kumar, A., Equeenuddin, S.M., Mishra, D.R., Acharya, B.C., 2016. Remote monitoring of sediment dynamics in a coastal lagoon: long-term spatio-temporal variability of suspended sediment in Chilika. Estuar. Coast. Shelf Sci. 170, 155-172.

Le, C.F., Li, Y.M., Zha, Y., Sun, D., Yin, B., 2009. Validation of a quasi-analytical algorithm for highly turbid eutrophic water of Meiliang bay and Taihu lake, China. IEEE Trans. Geosci. Remote Sens. 47 (8), 2492-2500.

Le, C., Hu, C., Cannizzaro, J., English, D., Muller-Karger, F., Lee, Z., 2013. Evaluation of chlorophyll-a remote sensing algorithms for an optically complex estuary. Remote Sens. Environ. 129, 75-89.

Lee, Z.P., Carder, K.L., Mobley, C.D., Steward, R.G., Patch, J.S., 1999. Hyperspectral remote sensing for shallow waters: 2. Deriving bottom depths and water properties by optimization. Appl. Opt. 38 (18), 3831-3843.

Lee, Z.P., Carder, K.L., Arnone, R.A., 2002. Deriving inherent optical properties from water color: a multiband quasi-analytical algorithm for optically deep waters. Appl. Opt. 41 (27), 5755-5772.

Lee, Z.P., Carder, K.L., 2004. Absorption spectrum of phytoplankton pigments derived from hyperspectral remote-sensing reflectance. Remote Sens. Environ. 89, 361-368.

Lee, Z.P., Lubac, B., Werdell, J., Arnone, R., 2009. An Update of the Quasi-analytical Algorithm (QAA_v5). IOCCG <http://www.ioccg.org/groups/Software_OCA/ QAA_v5.pdf >.

Lee, Z.P., Arnone, R., Hu, C., Werdell, PJ., Lubac, R., 2010. Uncertainties of optical parameters and their propagation in an analytical ocean color inversion algorithm. Appl. Opt. 49 (3), 369-381.

Lee, Z.P., 2014. An update of the quasi-analytical algorithm (QAA_v6). IOCCC 〈http://www.ioccg.org/groups/Software_OCA/QAA_v6_2014209.pdf>. 
Li, L., Li, L., Song, K., Li, Y., Tedesco, L.P., Shi, K., Li, Z., 2013. An inversion model for deriving inherent optical properties of inland waters: establishment, validation and application. Remote Sens. Environ. 135, 150-166.

Li, L., Li, L., Song, K., 2015. Remote sensing of freshwater cyanobacteria: an extended IOP inversion model of inland waters (IIMIW) for partitioning absorption coefficient and estimating phycocyanin. Remote Sens. Environ. 157, 9-23.

Matsushita, B., Yang, W., Yu, G., Oyama, Y., Yoshimura, K., Fukushima, T., 2015. A hybrid algorithm for estimating the chlorophyll-a concentration across different trophic states in Asian inland waters. ISPRS J. Photogramm. Remote Sens. 102, 28-37.

Mishra, S., Mishra, D.R., Shluchter, W.M., 2009. A novel algorithm for predicting phycocyanin concentrations in cyanobacteria: a proximal hyperspectral remote sensing approach. Remote Sens. 1 (4), 758-775.

Mishra, D.R., Mishra, S., 2010. Plume and bloom: effect of the Mississippi River diversion on the water quality of Lake Pontchartrain. Geocarto Int. 25 (7), 555 568.

Mishra, S., Mishra, D.R., 2012. Normalized difference chlorophyll index: a novel model for remote estimation of chlorophyll-a concentration in turbid productive waters. Remote Sens. Environ. 117, 394-406.

Mishra, S., Mishra, D.R., Lee, Z.P., Tucker, C.S., 2013. Quantifying cyanobacterial phycocyanin concentration in turbid productive waters: a quasi-analytical approach. Remote Sens. Environ. 133, 141-151.

Mishra, S., Mishra, D.R., Lee, Z.P., 2014. Bio-optical inversion in highly turbid and cyanobacteria-dominated waters. IEEE Trans. Geosci. Remote Sens. 52 (1), 375388.

Mobley, C.D., 1999. Estimation of the remote-sensing reflectance from abovesurface measurements. Appl. Opt. 38 (36), 7442-7455.

Morel, A., Prieur, L., 1977. Analysis of variations in ocean color. Limnol. Oceanogr. 22 (4), 709-722

Moses, W.J., Gitelson, A.A., Berdnikov, S., Saprygin, V., Povazhnyi, V., 2012. Operational MERIS-based NIR-red algorithms for estimating chlorophyll-a concentrations in coastal waters - the Azov Sea case study. Remote Sens. Environ. 121, 118-124.

Mueller, J.L., 2003. In-water radiometric profile measurements and data analysis protocols. In: Mueller, J.L., Fargion, G.S., McClain, C.R. (Eds.), Ocean Optics Protocols for Satellite Ocean Color Sensor Validation, Revision 4, Radiometric Measurements and Data Analysis Protocols, vol. III. NASA Goddard Space Flight Space Center, Greenbelt, Maryland, USA, pp. 7-20. NASA/TM-2003-21621/RevVol. III.

Odermatt, D., Gitelson, A., Brando, V.E., Schaepman, M., 2012. Review of constituent retrieval in optical deep and complex waters from satellite imagery. Remote Sens. Environ. 118, 116-126.

Ogashawara, I., Mishra, D.R., Mishra, S., Curtarelli, M.P., Stech, J.L., 2013. A performance review of reflectance based algorithms for predicting phycocyanin concentrations in inland waters. Remote Sens. 5, 4774-4798.

O’Reilly, J.E., Maritorena, S., Mitchell, B.G., Siegel, D.A., Carder, K.L., Garver, S.A. Kahru, M., McCalin, C., 1998. Ocean color chlorophyll algorithms for SeaWiFS. J. Geophys. Res. 103 (C11), 24937-24953.
Pope, R.M., Fry, E.S., 1997. Absorption spectrum (380-700 nm) of pure water. II. INTEGRATING cavity measurements. Appl. Opt. 36 (33), 8710-8723.

Rodrigues, T.P., Guimarães, U., Rotta, L., Watanabe, F., Alcântara, E., Imai, N., 2016. Delineamento amostral em reservatório utilizando imagens Landsat-8/OLI: um estudo de caso no reservatório de Nova Avanhandava (Estado de São Paulo). Boletim de Ciências Geodésicas 22 (2), 303-323.

Roesler, C.S., Perry, M.J., Carder, K.L., 1989. Modeling in situ phytoplankton absorption from total absorption spectra in productive inland marine waters. Limnol. Oceanogr. 34 (8), 1510-1523.

SeaBASS. SeaWiFS Bio-optical Archive and Storage System, 2015. NOMAD: NASA bio-Optical Marine Algorithm Dataset <http://seabass.gsfc.nasa.gov/wiki/ article.cgi? article=NOMAD>.

Simis, S.G.H., Peters, S.W.M., Gons, H.J., 2005. Remote sensing of the cyanobacterial pigment phycocyanin in turbid inland water. Limnol. Oceanogr. 50 (1), 237245.

Smith, R.C., Baker, K.S., 1981. Optical properties of the clearest natural waters (200$800 \mathrm{~nm})$. Appl. Opt. 20 (2), 177-184.

Tassan, S., Ferrari, G.M., 1995. An alternative approach to absorption measurements of aquatic particles retained on filters. Limnol. Oceanogr. 40 (8), 1358-1368.

Tassan, S., Ferrari, G.M., 1998. Measurement of light absorption by aquatic particles retained on filters: determination of the optical path length amplification by the ‘transmittance-reflectance' method. J. Plankton Res. 20 (9), 1699-1709.

Tassan, S., Ferrari, G.M., 2002. A sensitivity analysis of the "transmittancereflectance' method for measuring light absorption by aquatic particles. J. Plankton Res. 24 (8), 757-774.

Tundisi, J.G., Matsumura-Tundisi, T., Abe, D.S., 2008. The ecological dynamics of Barra Bonita (Tietê River, SP, Brazil) reservoir: implications for its biodiversity. Braz. J. Biol. 68, 1079-1098.

Vincent, R.K., Qin, X., McKay, R.M.L., Miner, J., Czajkowski, K., Savino, J., Bridgeman, T., 2004. Phycocyanin detection from LANDSAT TM data dor mapping cyanobacterial blooms in Lake Erie. Remote Sens. Environ. 89, 381-392.

Watanabe, F.S.Y., Alcântara, E., Rodrigues, T.W.P., Imai, N.N., Barbosa, C.C.F., Rotta, L. H.S., 2015. Estimation of chlorophyll-a concentration and the trophic state of the Barra Bonita hydroelectric reservoir using OLI/Landsat-8 images. Int. J. Environ. Res. Public Health 12, 10391-10417.

Weaver, E.C., Wrigley, R., 1994. Factors Affecting the Identification of Phytoplankton Groups by Means of Remote Sensing. NASA, Ames Research Center, Moffett Field, CA, USA.

Wei, J., Lee, Z.P., Lewis, M., Pahlevan, N., Ondrusek, M., Armstrong, R., 2015. Radiance transmittance measured at the ocean surface. Opt. Express 23 (9), 1182611837.

Yang, W., Matsushita, B., Chen, J., Yoshimura, K., Fukushima, T., 2013. Retrieval of inherent optical properties for turbid inland waters from remote-sensing reflectance. IEEE Trans. Geosci. Remote Sens. 51 (6), 3761-3773.

Zhu, W., Yu, Q., Tian, Y.Q., 2013. Uncertainty analysis of remote sensing of colored dissolved organic matter: evaluations and comparisons for three rivers in North America. ISPRS J. Photogramm. Remote Sens. 84, 12-22. 